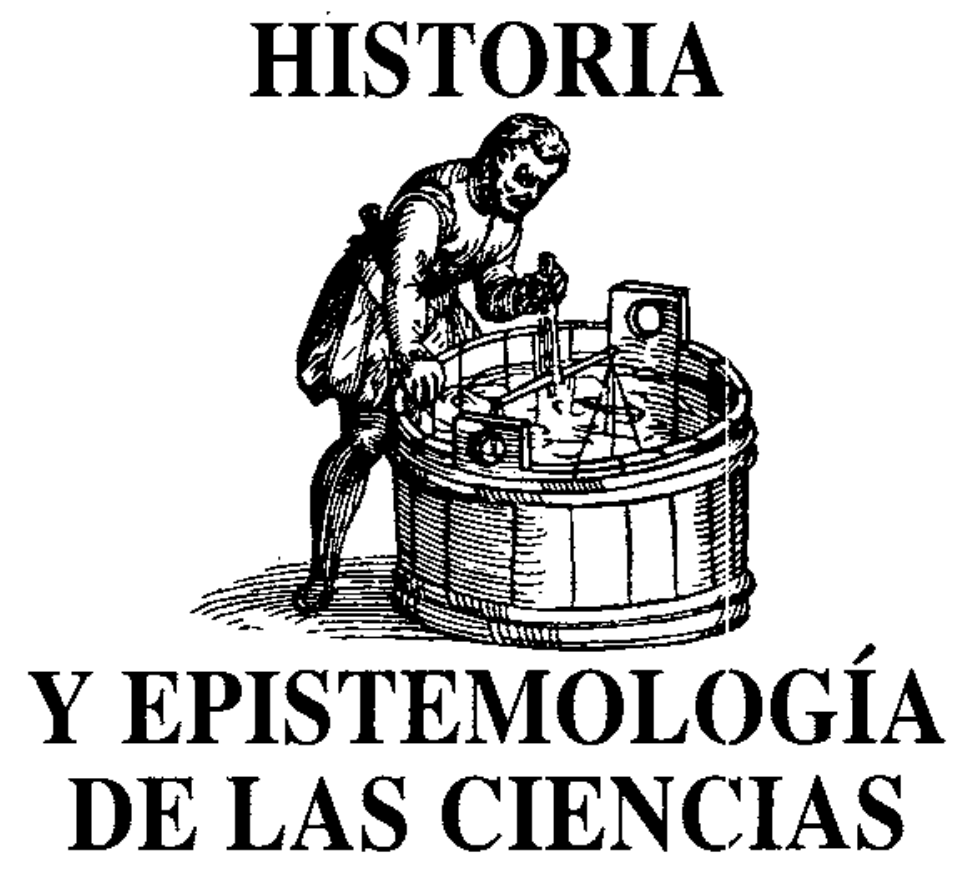

\title{
HISTORIA, FILOSOFÍA Y ENSEÑANZA DE LAS CIENCIAS: LA APROXIMACIÓN ACTUAL
}

MATTHEWS, M.R.

Education Department, Auckland University, Auckland, New Zealand.

\section{SUMMARY}

This paper traces the use of, and arguments for, the history and philosophy of science in school science courses. Specific attention is paid to the British National Curriculum proposals and "o the recommendations of the US Project 206 I curriculum guidelines. Some objections to the inclusion of historical material in science courses are outlined and answered. Mention is made of the Piagetian thesis that individual psychological development mirrors the development of concepts in the history of science. This introduces the topic of idealisation in science. Some significant instances are iternised where science education has, at its considerable cost, ignored work in philosophy of science. Arguments for the inclusion of the history and philosophy of science in science teacher education programmes are given. The paper finishes with a list of topical issues in present science education where collaboration between science teachers, historians, philosophers, and sociologists would be of considerable benefit.

\section{INTRODUCCIÓN}

En 1986 se publicó un trabajo titulado «Science Education and Philosophy of Science:Twenty-five Years of Mutually Exclusive Development» (Duschl 1986). En él se daba cuenta de que la evolución đe la enseñanza de las ciencias está enormemente separada de las disciplinas de historia y filosofía de la ciencia. En los últimos cinco años ha habido un acercamiento significativo entre estos campos. Cada vez más, la historia y la 
filosofía de la ciencia se van incorporando a la teoría y especialmente a la práctica de la enseñanza de las ciencias.

Dada la bien conocida crisis contemporánea de la enseñanza de las ciencias -reflejada en la huida del aula de ciencias, tanto de profesores como de estudiantes, y en la alarmante cifra de analfabetismo cientifico (Matthews 1988)- estas iniciativas son oportunas. La American National Science Foundation denunció que «los programas nacionales de ciencias, matemáticas y tecnología en la educación secundaria habían decaído en calidad y extension, hasta tal punto que ya no respondian a las necesidades nacionales. Un recurso singular americano ha sido agotado» (Heilbron 1987, p. 556). La historia, filosofía y sociología de la ciencia no tienen todas las soluciones para esta crisis pero sí tienen algunas respuestas: pueden humanizar las ciencias y acercarlas más a los intereses personales, éticos, culturales y políticos; pueden hacer Ias clases más estimulantes y reflexivas, incrementando así las capacidades del pensamiento crítico; pueden contribuir a una comprensión mayor de los contenidos científicos; pueden contribuir un poco a superar el «mar de sinsertidos» en que un comentarista dijo se habian engolfado las clases de ciencias, donde se recitaban fórmulas y ecuaciones, pero donde pocos conocían su significado; pueden mejorar la formación del profesorado contribuyendo al desarrollo de una epistemología de la ciencia más rica y más auténtica, esto es, a un mejor conocimiento de la estructura de la ciencia y su lugar en el marco intelectual de las cosas. Este último punto es el de partida de la clase de disciplina cognitiva que Schulman (1987), y antes que él, pero olvidado durante mucho tiempo, Scheffler (1970), había pedido que promovieran los programas de formación del profesorado.

Hay algunos aspectos destacables en este acercamiento. El más significativo, con mucho, es la inclusión de contenidos de historia y filosofía de la ciencia en varios currículos educativos nacionales. Así sucede en el currículo nacional de ciencias de Inglaterra y Gales, en las recomendaciones para las ciencias en la enseñanza secundaria en el proyecto americano 2061 , en el currículo educativo nacional danés y en los materiales curriculares del PLON holandés. No se trata de la inclusión de HFC (historia y filosofía de la ciencia) como un ítem más de los contenidos, sino más bien la incorporación general de temas de HFC a las expectativas de los contenidos y enseñanzas de los currículos. Los currículos de ciencias han incluido normalmente una sección titulada "The Nature of Science». Ahora se presta más atención a estas secciones y cada vez más se reconoce que Ia historia, filosofía y sociología de la ciencia contribuyen a una comprensión mejor, más completa y más rica de los asuntos planteados en esas secciones. Una mayor apertura pará la contribución histórica y filosófica a la enseñanza de las ciencias se da en la amplia difusión de programas de ciencia, técnica y sociedad (CTS) tanto en la secundaria como en universidades. Estos avances tienen significativas implicaciones en la formación del profesorado ${ }^{2}$.

Hay otras muestras o indicios de este acercamiento. Uno de ellos ha sido la celebracion del primer congreso internacional sobre «HFS and Science Teaching» en la Universidad del estado de Florida, en noviembre de $1989^{3}$. Otro, la serie de congresos patrocinados por la European Physical Society sobre «History of Physics and Physics Teaching» en Pavia (1983), Munich (1986), París (1988), Cambridge (1990) y Madrid (1992) . Untercero ha sido el congreso organizado por la British Society for the History of Science sobre «History of Science and Science Teaching» celebradoen la Universidad de Oxford en 1987 (Shortland y Warick 1989). Estas actividades han producido cerca de 350 trabajos educativos sobre la materia, así como una cantidad considerable de material de enseñanza tratado histórica y filosóficamente. Adcmás, la American National Science Foundation ha comenzado ahora dos programas para introducir la HFC en la enseñanza de las ciencias en escuelas e institutos. Algunos programas de formación de profesores de ciencias en EEUU han hecho materia obligatoria la HFC. El estado de Florida ha hecho que sea necesario completar un curso de HFC para poder acreditarse como profesor de ciencias.

Los defensores de la HFC en Ia enseñanza de las ciencias están defendiendo, de algún modo, una versión «contextualizada» de la enseñanza de las ciencias. Es decir, una enseñanza de las ciencias que enseñe ciencias en su contexto social, histórico, filosófico, ético y tecnológico. En parte, esto es una reelaboración del viejo argumento: la enseñanza de las ciencias debería ser una enseñanza sobre la ciencia, así como en la ciencia. Usando la terminología del currículo nacional británico, los estudiantes de ciencias en la secundaria deberían aprender algo sobre «la naturaleza de la ciencia», así como el contenido de la ciencia propiamente dicha.

Las discusiones o acercamientos están repitiendo de maneras diferentes las recomendaciones que comenzaron con Mach ${ }^{5}$ al final del siglo XIX y fueron repetidas por Duhem a principios del xx. Tales recomendaciones han sido hechas nuevamente por autores como Nunn, Conant, Holton, Robinson, Schwab, Martin y Wagenstein. Pueden consultarse en numerosos informes británicos y americanos.

\section{LA REFORMA DE LOS CURRÍCULOS}

Cuando se publica cualquier artículo sobre HFC y ensefranza de las ciencias, vale la pena comentar el nuevo currículo nacional británico de ciencias y el proyecto americano AAAS 2061, porque muestran claramente las implicaciones del citado acercamiento en el aula y en los programas. El primero es un curso ya implantado; el segundo es un conjunto de propuestas comprensivas, planificado hace tiempo para un nuevo currículo de ciencias americano.

La introducción del British National Curriculum Council en el núcleo de HFC del curso (que constituye casi el $5 \%$ del programa total), dice que los alumnos deberían mejorar su conocimiento y comprensión de las formas con que cambian las ideas cientificas a través del ticmpo y cómo la naturaleza de estas ideas y los usos a que se aplican son afectados por los contextos social, moral, 
espiritual y cultural donde se desarrollan (NCC 1988, p. 113).

Como un ejemplo del tipo de habilidades y de comprensión que el NCC intenta promover en el nuevo currículo, podrían ser instructivas sus manifestaciones sobre las aptitudes de los estudiantes de 4-16 años. Deberían ser capaces de:

- distinguir entre exigencias y argumentos basados en evidencias y datos científicos y los que no lo están;

- considerar cómo el desarrollo de una idea o teoría científica concreta se relaciona con su contexto histórico y cuitural, incluyendo el espiritual y el moral;

- estudiar ejemplos de controversias científicas y las formas en que las ideas científicas han cambiado.

La American Association for the Advancement of Science (AAAS) propuso en 1985 un amplio estudio nacional, proyecto 2061, encaminado a elevar el interés por la ciencia en escuelas e institutos. En 1989, después de cuatro años de deliberaciones y consultas, fueron publicadas sus recomendaciones en un informe titulado «Science for All Americans》 (AAAS 1989). El proyecto 2061 estaba al margen de las deliberaciones del británico NCC, pero hay una coincidencia considerable respecto a la necesidad de que las ciencias en la primaria y en la secundaria estén más contextualizadas, sean más históricas y más filosóficas o reflexivas.

«Science for All Americans» consta de doce capítulos con las recomendaciones para las ciencias en escuelas e institutos del National Council On Science and Technology Education. El capitulo uno es sobre «The Nature of Science». Trata de las discusiones sobre objetividad; mutabilidad de la ciencia; posibles formas de distinguir la ciencia de la seudociencia; la evidencia y su relación con la teoría de la justificación; el método científico; explicación y predicción; ética, influencia social y la organización social de la ciencia. El propósito es que esos temas sean desarrollados y discutidos dentro de los cursos de ciencias y que los alumnos que acaban los estudios de ciencias primarios y secundarios sepan algo de ellos; no se trata de que dichos temas sean añadidos a los cursos de ciencias y que HFC sustituya conocimientos de contenido científico.

La introducción al capítulo diez sobre "Historical Perspective» dice: «Entre las recomendaciones hay dos razones principales para incluir algún conocimiento de historia. Una razón es que las generalizaciones sobre cómo procede la empresa científica quedarían vacías sin ejcmplos concretos». Continúa diciendo: «Ũna segunda razón es que algunos episodios en la historia del esfuerzo científico son de importancia capital para nuestra herencia cultural. Tales episodios incluyen, sin duda, el papel de Galileo en el cambio de nuestra percepción del lugar que ocupamos en el universo.»

El artículo dedica página y media al episodio de Galileo, «Displacing the Earth from the Center of the Universe». Tiene un tratamiento documentado y sugestivo sobre evidencias astronómicas, el papel de la percepción sensorial, modelos matemáticos, realismo e instrumentalismo, metafísica, tienología, retórica y teología. Trata otros episodios históricos de una forma similar.

Tanto en las propuestas de los currículos británicos como en las del americano, no se pretende que una retahila de conclusiones sobre ciencia sea reemplazada por una retahila de conclusiones sobre HFC. Nadie espera que los niños resuelvan el debate realismo-instrumentalismo, ni que aprendan, como si fuera el catecismo, que habia quince razones por las que Galileo tenía razón y los cardenales estaban equivocados. Lo que se espera realmente es que capten algo de los aspectos intelectuales que están en juego en estos asuntos, que capten que ray preguntas a hacer y que comiencen a pensar no sólo en las respuestas, sino sobre to que podría considerarse como respuesta y qué tipos de evidencias pueden respaldar nuestras respuestas.

La conversión de los proyectos curriculares, en realidad, en el aula reşuerirá nuevas guías de evaluación y práctica, nuevos materiales de enseñanza y, sobre todo, Ia inclusión de cursos de HFS apropiados a los cursos de formación de: profesores. También requerirá, como explícitamente se afirma en las propuestas del proyecto 2061, que se rebajen los contenidos de los currículos «hinchados $y$ desnutridos» (AAAS 1989, p. 14). Un estudio del programa escolar de biología del estado de Nueva York muestra la dimensión de este problema: a los profesores se les exige introducir 1440 nuevos términos y conceptos científicos en un año (Swift 1988). Ahora se aprecia mejo:: Io que Mach defendfa hace un siglo: «Creo que la cantididd de materia necesaria para una enseñanza útil [...] es muy pequeña [...] No conozco nada más terrible que las pobres criaturas que han aprendido demasiado [...] Lo que han adquirido es una maraña de pensamientos demasiado débil para proporcionar soportes seguros, pero lo bastante complicada como para producir confusión» (Mach 1943, p. 366).

\section{LA HISTORIA EN EL CURRÍCULO DE CIENCIAS}

En Gran Bretaña ha habido una larga, aunque limitada e irregular, tradición de incorporar la historia de la ciencia a la enseñanza de las ciencias. Esto ha sido bien documentado por Edgar Jenkins $(1989,1990)$ y W. J. Sherratt (1982, 1983). Según Jenkins, la primera referencia clara data nada menos que de 1855 , en el discurso presidencial del duque de: Argyll en la British Association for the Advancemert of Science, donde dijo:»Lo que buscamos en la formación de los jovenes no es tanto los meros resultados como los métodos y, sobre todo, la historia de la ciencia» (Jenkins 1989, p. 19). La BAAS repitió estos llamamientos en su conferencia de 1917 donde remarcó que la historia de la ciencia proporcionaba una solución a esa barreri artificial entre los estudios literarios y cientificos, que establecen los horarios escolares (Jenkins 1989, p. 19). Mach y otros influidos por él también argumentaron que para comprender un concepto teórico era necesario comprender su desarrollo histórico; el conocimiento era necesariamente histórico. Mach en su obra yá clásica de 1883 decía que: 
«La investigación histórica del đesarrollo de la ciencia es de lo más necesario, salvo que los principios atesorados en ella se conviertan en un sistema de preceptos a medio entender, o lo que es peor, en un sistema de prejuicios. La investigación histórica no sólo promueve la comprensión de lo actual, sino que también nos proporciona nuevas posibilidıtdes» (Mach 1883-1960, p. 316) ${ }^{6}$.

Nunn y olros educadores con mentalidad histórica argumentaron lo mismo en el período entre guerras. Varios tribunales de exámenes prepararon diferentes cursos de historia de la ciencia, pero hacia 1980 el número de candidatos que se presentaba era exiguo. Con anterioridad al Currículo Nacional, la historia de la ciencia encontró un lugar en los programas Nuffield y en los cursos SISCON y SATIS, recientemente introducidos.

Entre 1970 y 1980, la British Association for Science Education, en algunos de sus informes (Alternatives for Science Education 1979, Education through Science 1981), recomendaba con insistencia la incorporación de más material histórico y filosófico en el currículo de ciencias. Un problema que reconocian era que los profesores no estaban adecuadamente preparados para ensenar esta ciencia contextual. En su informe de 1963 (The Training of Graduate Teachers), se dice, de los profesores graduados, que «muchos actúan y piensan científicamente, como resultado de su preparación, pero carecen del conocimiento de la naturaleza y objetivos básicos de la ciencia) (p. 13). Este punto fue tratado nuevamente en un artículo de 1981 sobre el lugar de la filosofía de la ciencia eri la formación del profesor de ciencias británico (Manuel 1981).

En Estados Unidos, después de la segunda guerra mundial, la historia de la ciencia figuró de forma destacada en cursos universitarios de ciencias para no científicos. La influencia del presidente de la Universidad de Harvard, Jantes B. Conant, fue dominante puesto que sus aproximaciones históricas -los casos-estudio- fueron ampliamente adoptados. Los desarrolló mientras estaba al cargo de la educación general de estudiantes de bachillerato en Harvard y los popularizó en una serie de informes oficiales y en besisellers de bolsillo, siendo el más conocido Understanding Science: An Historical Approach (1947). Sus dos volúmenes Harvard case Histories in Experimental Science (1957) se convirtieron en el libro de texto de muchos cursos de ciencias.

La influencia de Conant no puede ser sobreestimada. Kuhn dice que Conant fue "quien primero me introdujo en la historia de la ciencia y así inicié la transformación de mi corcepción de la naturaleza del progreso cientificon (Kuhn 1970 p. XI); la relación de Kuhn con Conant está relatada en Merton (1977, pp. 81-89). Las subsiguientes transformaciones postkuhnianas en historia y filosofía de la ciencia son bien conocidas. Gerald Holton admite algo similary, como Kuhn, dice que la experiencia de ser un científico que, de repente, tuvo que impartir un curso en Harvard de educación general, montado en torno a la historia de la ciencia, fue una experiencia paulina. Leo Klopfer adoptó los casos-estudio de Harvard para su uso en escuelas e institutos y fueron probados con un éxito considerable (Klopfer y Cooley 1963).
Gerald Hoiton fue consiguientemente cooperador al desarroilar a comienzos de los años 60 , con Stephen Brush, Fletcher Watson, James Rutherford y otros, el Harvard Project Physics Course para la enseñanza secundaria. La conexión Conant-Harvard continuóa a lo largo de los años 80 con el nombramiento de James Rutherford como director del programa Proyecto 2061 de la AAAS.

Bernard Cohen, el destacado historiador de la ciencia de Harvard, también defendió la introđucción de material histórico en los programas universitarios de ciencias. Organizó un simposio sobre este asunto en la conferencia anual de 1950 de la American Association of Physics Teachers. Contribuyó con la conferencia principal «A Sense of History in Science» (1950), donde abordó algunos tratamientos convencionales de episodios históricos e indicó las numerosas inexactitudes cometidas en ellos. Extendió la distorsion de datos históricos hasta la física del siglo xx. Citó las referencias comunes, pero equivocadas, de los experimentos de J. J. Thompson para determinar la relación e/m. Cohen recomienda un mejor conocimiento de la historia de la ciencia, aconseja a los profesores que intenten escribir historia y sostiene que un sentido histórico hará las lecciones «más ricas y más profundas y de un mayor interés para [...] los estudiantes».

Sin embargo, y esto está bien documentado (DuschI 1985), las grandes reformas curriculares de ciencias de los años 60 se realizaron, en gran parte, sin la participación de historiadores ni filósofos de la ciencia (podría decirse que en muchas de ellas se procedió sin contar siquiera con los maestros de escuela y profesores). Hubo dos excepciones notables. Una fue el ya mencionado Harvard Project Physics Course; Ia otra, las versiones del American Biological Science Curriculum Study (BSCS).

El Harvard Project Physics Course que en su mejor momento fue seguido por casi el $15 \%$ de los estudiantes de física de secundaria de los Estados Unidos, ha sido el currículo educativo de ciencias basado en principios históricos y relacionado con la dimensión cultural y filosófica de la ciencia que más ampliamente se ha implantado en Estados Unidos. Su éxito en mantener estudiantes, involucrar mujeres en los cursos de ciencias, desarrollar habilidades de pensamiento crítico y alcanzar récords en la superación de exámenes, ha servido de apoyo para los actuales defensores de HFC. Este testimonioestá reseñadoen Aikenhead (1974), Holton (1978), Russell (1981), Brush (1989) y en el simposio sobre proyectos de física en The Physics Teacher, 5 (2), 1967. Las enseñanzas sacadas de los fallos de los proyectos de f́́sica son también instructivas, en particular el fallo esperado para familiarizar adecuadamente a los profesores con HFC, de manera que pudieran enfrentarse con el curriculo de forma abierta y crítica. Esto ha sido identificado ya como un problema serio para la implementación del componente sobre la «naturaleza de la ciencia» en el British National Curriculum (NCC 1988, p. 21).

El BSCS se fundamentó en las ideas del educadorbiólogo-filósofo J. J. Schawb ${ }^{7}$, quien promovió la doctrina pedagógica de la «ciencia como indagación». Schawb escribió el Teachers'Handbook para el currículo del BSCS 
y en él defendía la aproximación histórica diciendo que «la esencia de la enseñanza de la ciencia como indagación podría mostrar algunas conclusiones científicas en la forma en que surgen y son comprobadas [...] incluyendo también un tratamiento adecuado de las dudas y limitaciones de la ciencias (1963, p. 4I). La historia también es defendida porque «concierne al hombre y a los sucesos más que a las concepciones mismas. Existe un lado humano en la indagación» (1963, p. 42).

A principios de los años 60 la International Comission on Physics Education defendió la utilidad de la historia de la física en la enseñanza de la física. En 1970 tuvo lugar un simposio sobre este asunto en el MIT, bajo la dirección de Stephen Brush y Allen King. Las actas fueron publicadas (Brush y King 1972), y constituyen un auténtico reto, según Klein, para cualquier proyecto sobre utilización de la historia de la física en la enseñanza de la física. Esto será comentado en la sección siguiente.

En los años 70 la American Physical Society estableció una sección de Historia de la Física, y al mismo tiempo ia History of Science Society estableció un Comité de Educación. Ambos han continuado su actividad en materia educativa. Stephen Brush ha influido en ellos, produciendo numerosos estudios históricos para su uso en el aula ${ }^{\mathrm{g}}$.

La historia de la química en US ha sido siempre más marginal para la pedagogía que la historia de la física o de la biología. Ha habido numerosas propuestas para que la historia de la química se incluya en la enseñanza de la química; esto está bien documentado en Kaufmann (1989).

En otros países, la historia de la ciencia ha tenido la misma variedad de incidentes en la pedagogía de la ciencia. Breves informes de las experiencias europeas se pueden encontrar en las publicaciones de Thomsen (1986). Teichmann en el Deutsches Museum de Munich ha reproducido experimentos históricos y propuesto notas e instrucciones para profesores; un esfuerzo similar ha realizado Bevilacqua y colaboradores en la Universidad de Pavía. Krasilchik (1990) comenta una interesante aproximación al tema en Brasil; Tamir (1989) comenta la situación en Israel.

\section{LA HISTORIA BAJO CRÍTICA}

La tradición contextualista afirma que la historia de la ciencia mejora la enseñanza de las ciencias porque: 1) motiva e interesa a los alumnos; 2) humaniza los contenidos; 3) proporciona una mejor comprensión de Ios conceptos científicos mostrando su desarrollo y perfeccionamiento; 4) tiene un valor intrínseco la comprensión de ciertos episodios cruciales en la historia de la ciencia: revolución científica, darwinismo, etc; 5) demuestra que la ciencia es mutable y cambiante y que, en consecuencia, el conocimiento científico actual es susceptible de ser transformado; lo que 6) de esta manera, combate la ideología cientifista; y finalmente 7), la historia permite un conocimiento más rico del método científico y muestra las pautas del cambio de la metodología aceptada.
En 1970, en la antes mencionada conferencia MIT, estas justificacion:s sobre la historia fueron atacadas desde dos frentes: $\epsilon$ n primer lugar, se dijo que la única historia posible en curso de ciencias era pseudohistoria; en se. gundo lugar, se dijo que exponer la historia de la ciencia debilitaba las convicciones científicas necesarias para conseguir ccn éxito el aprendizaje de las ciencias. El primer caso fue argumentado por Martin Klein (1972); el segundo fue, en parte, favorecido por el análisis hecho en el clásico de Thomas Kuhn The Structure of Scientific Revolutions i $1{ }^{\text {a }}$ ed. $1962,2^{a}$ ed. 1970).

$\mathrm{El}$ argumentc de Klein era que los profesores de ciencias (particularminte los de física) seleccionan y usan materiales histórisos más allá de los propósitos científicos o pedagógicos

«Estamos, en otras palabras, planificando seleccionar, organizar y presentar estos materiales históricos no históricamente, quizás incluso antihistóricamente. Esta es una empresa muy azarosa, si estamos preocupados tanto por la integridad y calidad de la historia que enseñamos como por la física» (Klein 1972, p. 12).

\section{Continúa diciendo:}

«Una razón cue dificulta conseguir que la historia de la física sirva a las necesidades de la enseñanza de la física es la diferencia esencial entre las perspectivas del físico y del historiador [...] Así, es difícil imaginar la combinación entre la rica complejidad de hechos, a los que el historiador aspira, con la visión simple y precisa que el f́́sico buscan (Klein 1972, p. 16).

Su conclusićn es que, si una buena enseñanza de la ciencia está históricamente informada, entonces sólo tendrá la posibilidad de usar mala historia. Prefiere prescindir de la historia ante una mala historia.

Whitaker (1979) fue aún más lejos en un artículo titulado «History and Quasi-history in Physics Education». Su preocupación era identificar las construcciones preferentes de historia pira adecuar no sólo los fines pedagógicos sino los fines de ideología científica también, o la visión de la ciencia sostenida por el escritor. Estos casos abundan en los lib:os de texto. Uno que ha sido muy discutido es la explicación muy difundida de cómo la teoría de la relatividad especial de Einstein fue suscitada por el resultado nulo del experimento de Michelson-Morley. Éste es un mito de inspiración popperiana. Otro mito es la visión generalizada de que el postulado del fotón de Einstein sigu.ó a los experimentos del efecto fotoeléctrico, mejor que precedió a los experimentos. Entre otros innumerables ejemplos, hay uno en las primeras páginas del libro de exto del PSSC que describe el descubrimiento de $G$ ałilleo de la ley de isocronía del péndulo cronometranto el balanceo del candelabro de la iglesia de Pisa. Esta rludosa explicación es utilizada por el PSSC como el vercladero modelo de metodología científica. Los problemas que plantea se comentan en Matthews (1987).

Whitaker hat la de la «casi historia» como el «resultado de numerosos: libros de autores que han sentido la nece- 
sidad de dar vida a sus explicaciones de [estos episodios] con un poco de contenido histórico, pero que de hecho han reescrito la historia acomodándola paso a paso con la física» (Whitaker 1979, p. 109).

«Casi historia» no es exactamente la pseudohistoria de Klcin, o historia simplificada, donde es probable que se produzcan fallos por omisión, o donde el relato podría quedar por debajo del sublime dicho: «la verdad, toda la verdad y nada más que la verdad». Esto es parecido a las «reconstrucciones racionales» de la historia de Lakatos (1978), donde la historia está escrita para apoyar una versión particular de la metodología científica y donde las figuras históricas están pintadas con los colores de la metodología ortodoxa común.

"Casi historia» es un asunto complejo. Sabemos que la objetividad en la historia es, en principio, imposible: la historia no se presenta tal cual es a los ojos del espectador; tiene que ser construida. Los materiales y fuentes han de ser seleccionados; las cuestiones han de ser formuladas; han de tomarse decisiones sobre las contribuciones relevantes de factores internos y externos en el cambio científico. Todos estos aspectos están influidos por las opiniones; sociales, nacionales, psicológicas y religiosas del historiador. Y lo que es más importante, están influidas por la teoría de la ciencia, o la filosofía de la ciencia, sostenida por el historiador. Así como la teoría de un científico afecta a cómo ve, selecciona y trabaja su material, también la teoría de un historiador afecta a cómo ve, selecciona y trabaja el suyo. Como mucha gente ha dicho, si la filosofía de la ciencia está vacía sin historia de la ciencia, la historia de la ciencia sin filosofía de la ciencia está ciega?

La historia de la interpretación de los logros y la metodología de Galileo y las traducciones de sus trabajos ilustran el problema de cómo la teoría afecta la forma en que los hechos y documentos históricos son considerados. Para los filósofos y científicos del siglo xIx, Galiteo era un inductivista y empirista. William Whewell dice de él que "quizá tenía una inclinación predominante hacia Ios hechos, y no sentía tanto como otros personajes de su tiempo la necesidad de reducirlos a ideas" $(1840,1947$, p. 220). David Brewster en 1830 lo veía como una figura baconiana y afirmaba que se podían encontrar en sus trabajos «los pricipios de la filosofía inductiva» (Finocchiaro 1980 , p. 152). Cuando el positivismo estaba en auge, se presentaba a Galileo en los textos como un positivista. Mach dice que «Galileo no nos suministro una teoría de la caída de los cuerpos, sino que investigó, sin ninguna opinion preconcebiđa, el hecho real de la caída» (Mach 1883,1960 , p. 167). En Ios años 30 cayó una bomba sobre la interpretación empirista de Galileo cuando Alexandre Koyré anunció que Galíleo era realmente platónico $(1939,1943)$. La historia de la interpretación platónica ha sido comentada en McTighe (1967). La visión racionalista de Galileo de William Shea (1972) tiene mucho en común con la de Koyré. Pero empirismo y racionalismo no agotan todo el campo interpretativo. William Wallace ha escrito una serie de estudios situando a Galileo en la tradición escolástico-aristotélica (1981, 1984); un punto de vista sugerido en el estudio de la Escuela die Padua por Randall (1940). La imagen que surge de los muchos artículos y libros de Stillman Drake $(1978,1980)$ es la de un Galilco como paciente experimentalista. Es bien conocida la reciente interpretación anarquista, o dadaísta, de Galileo por Paul Feyerabend: para él, Galileo es la pieza clave en contra de la primacía de un único método científico (1975).

Un comentarista de los trabajos comparados de Koyré y Drake escribió:

«El Galileo de Koyré parecía habitar un mundo enormemente filosófico de platonismo, copernicanismo, racionalismo y experimentos mentales. Por otra parte, el Galileo de Drake es más activo y menos contemplativo [...] un agudo observador, experimentador e inventor [...] Estas considerables diferencias en las conclusiones de Drake y Koyré proceden, en gran medida, de Ias diferencias de sus propios estilos de estudio e investigación (Mac Lachlan 1990, p. 124).

Que una interpretación de un texto o de un hecho refleja las opiniones de la época o del intérprete ya fue señalado al final del siglo xIX por estudiosos bíblicos, la escuela crítica de Renan y otros. La monumental obra de Albert Schweitzer The Quest for the Historical Jesus (1910), después de comentar la historia de las imágenes y teologías sobre Jesús, concluyó que «cada época y cada individuo construyen vidas de Jesús a su propia imagen». Una conclusión reproducida sesenta años más tarde es cuando el evangelísta americano Billy Graham afirmó que «Jesús fue el más grande manager general que el mundo ha conocido».

La trađucción de la obra de Galileo estuvo también comprensiblemente afectada por los presupuestos de su traductor. Bernard Cohen ha llamado la atención sobre la totalmente gratuita inclusión, como experimento, en Discourse on Two New Sciences traducido por Crew y Salvio (1977), donde Galileo dice que ha descubierto algunas propiedades del movimiento desconocidas hasta entonces. Peter Machamer (1978) cita que la traducción de Drake del mismo trabajo interpreta la urazón» de Galileo como «norma», cuando el texto original da a entender «causa formal». Como Drake creyó que Galileo había abandonado la noción de causa final, no es sorprendente que el término no haya sido traducido así.

El problema es, por supuesto, más profundo que simplemente la interpretación que sigue a la percepción. Bacon, hace mucho tiempo, en su discusion sobre los «f́dolos de la mente» reconocía cómo supuestos personales y culturales, incluyendo el lenguaje, afectan la imaginación y el conocimiento. Bacon dio un consejo empirista, diciendo que deberíamos reducir al mínimo el conjunto de esas influencias y ver el mundo cómo es realmente. Ahora este consejo se reconoce como algo muy simplista: no sólo la literatura, la historia y la política, sino también la ciencia natural tienen su problema hermenéutico, una consideración defendida por Toulmin (1983) y Markus (1987). El sin duda ortodoxo historiador de la ciencia, A.C.Crombie, en su trabajo «Philosophical Presuppositions and Shifting Interpretations of Galiteo» (1981), reconoce la necesidad de una interpretación hermenéutica de la historiografía de la ciencia. 
El segundo cargo aducido contra la historia de la ciencia genuina, en los cursos de ciencias, era que puede minar el espíritu del científico neófito. Este cargo fue interpuesto por Thomas Kuhn, entre otros. En un ensayo de 1959 sobre educación científica y sus efectos psicológicos e intelectuales decía:

«La característica más destacada de esta educación es que, con una extensión inusual en otros campos creativos, está totalmente dirigida a través de los libros de tex to [...] no se anima a los estudiantes de ciencias a leer los clásicos de historia de sus respectivos campos, trabajos en los que podrian descubrir otras formas de ver los problemas discutidos en los libros de tex to [...] queda como una iniciación dogmática en una tradición preestablecida» (Kuhn 1959, 1977, pp. 228-229).

Khun dice que esta iniciación es necesaria porque «ninguna parte de la ciencia ha progresado tanto o tan rápidamente antes de que esta educación convergente [...] fuera posible» (p. 237). Kuhn adelantó estas ideas sobre la virtud de una enseñanza conformista de la ciencia, en su enormemente influyente The Structure of Scientific Revolutions, donde dice que, en la clase de ciencias, Ia historia de la ciencia debería ser distorsionada y los científicos anteriores deberían ser presentados trabajando los mismos problemas que los científicos modernos (1970, p. 138). Esta distorsión pretende conseguir que el aprendiz de científico se sienta parte de una tradición de búsqueda exitosa de la verdad: «... los libros de texto comienzan así por truncar el sentido del científico sobre la historia de su disciplina, proporcionando un sutitutivo de lo que en ellos se ha eliminado» (p. 137).

Stephen Brush amplió el cargo de Kuhn en su «Should the History of Science be Rated X?" (1974). En él sugiere que la historia de la ciencia podría tener una mala influencia en los estudiantes, porque socava tanto las certezas del dogma científico como la utilidad para mantener el entusiasmo de los aprendices. Él sugiere, quizá hablando burlonamente, que la historia de la ciencia debería restringirse a audiencias cientfficas maduras.

Las objeciones de Kuhn pueden remontarse a las reacciones ante la concepción instrumentalista de la ciencia de Poincaré, a principios de este siglo. Heilbron cuenta que el presidente de la British Association for the Advancement of Science, en 1901, dijo, sobre Ia teoría de la ciencia de Poincaré, que, «si la confianza en que sus métodos son armas con las que él puede abrirse paso hacia la verdad fue tomada de los instrumentos científicos, la paralización de éstos empeñados en una tarea sin esperanza recaería sobre él» (Heilbron 1983, p. 178).

Las objeciones de Klein-Kuhn son serias, pero sus puntos principales pueden adaptarse sin desechar la historia en los cursos de ciencias. En pedagogía, como en la mayor parte de las cosas, la materia necesita ser frecuentemente simplificada. Esto es verdad tanto para la historia de Ia ciencia como para la economía o para la ciencia misma. El hecho de que se simplifique la historia de Ia ciencia no es un golpe concluyente contra ella. La tarea pedagógica es producir una historia simplificada que ilustre la materia, pero sin que sea una caricatura del proceso historico. La simplificación estará adecuada a la edad del grupo al que se enseña y al currículo que se presenta. La historia y la ciencia pueden complicarse a medida que la situación educativa lo exija. El problema de una gran distorsión se aborda mejor con una buena presentación de $\mathrm{HFC}$ en la formación del profesorado antes y durante el ejercicio activo: la mayor parte de las distorsiones se hacen de buena fe. El problema hermenéutico de lá. interpretación de la historia de la ciencia, lejos de ser una barrera o un impedimento para el uso de la historia, puede ser la ocasión para introducir a los estudiantes tn los aspectos significativos sobre cómo leer textos e interpretar hechos, en los complejos problemas de la significación: los estudiantes saben, desde su vida cotidiana, cómo la gente ve las cosas de forma diferente, la historia de la ciencia es un vehículo natural para ilustrar cómo este hecho sucede en la ciencia misma.

Un pastel se prueba comiéndolo. Los estudios históricos han mostradij su valor para los profesores de ciencias: Arons (1988), Pumfrey (1989), Bevilacqua (1990) y los Proceedings de $1983,1986,1988$ y 1992 de las conferencias de lá European Physical Society contienen la clase de historia de la ciencia aplicada y pedagógicamemte útil que $\mathrm{H}$ illoron recomienda que sea hecha colaborando historiadores y educadores. No hay evidencia de que tales aproximaciones hayan disminuido el conocimiento cientifico; puede haber disminuido la convicción de ciertos científicos, pero eso puede no ser malo. EI éxito del Harvard Project Physics es un contraejemplo de las peocupaciones de Kuhn-Brush: en él se demuestra que una buena educación científica es, de hecho, posible sin adoctrinamiento (Siegel 1979).

\section{HISTORIA DE LA CIENCIA Y PSICOLOGÍA DEL APRENDIZAJE}

Una parte significativa de las publicaciones recientes sobre $\mathrm{HFC}$ y enseñanza de las ciencias se ha ocupado de la asociación de la historia de Ia ciencia y la psicología del aprendizaje. Más específicamente, ¿en qué forma se influyen mut uamente el desarrollo cognitvo individual y el proceso clel desarrollo histórico conceptual? Este tema ha tenicio una larga historia; ha recibido un nuevo impulso de liss rápidamente extendidas teorías cognitivas de la ciercia, en las que los conceptos y métodos de la ciencia colsnitiva (del conocimiento) han sido usados para estudiaj los procesos y la historia de la ciencia (Giere 1987, Jung 1986, Nessersian 1989).

Estos temas fueron explorados por primera vez en $T$ the Phenomenoligy of Mind de Hegel (1806), donde, en las primeras páginas, la idea de una dialéctica de las teorías del conocimjento se conecta con una dialéctica de las formas históricas de consciencia. Es decir, así como la epistemología (una característica objetiva) se desarrolla, la experiencia (una característica subjetiva) sobre la que se bas a el conocimiento cambia. Mach y Duhem repiten esta idea a finales deI siglo xIx. En nuestro siglo, este punto de vista ha tenido la exposición más influyente en los escritos de Jean Piaget; ciertamente esto refuerza su idea toral del desarrollo cognitivo. 
En un pasaje muchas veces citado de Genetic Epistemology (1970), dice Piaget:

«La hipótesis fundamental de la epistemología genética es que existe un paralelismo entre el progreso realizado en la organización lógica y racional del pensamiento (historia de la ciencia) y los correspondientes procesos psicológicos formativos» (p. 13).

El último y más extenso argumento de Piaget para su tesis se encuentra en Psychogenesis and the History of Science (1989). Sin embargo, ia naturaleza del «paralelismo» está, en cierto modo, poco clara en Piaget, y menos aún en quienes se inspiraron en él: las posiciones varian desde la analogía hasta el isomorfismo débil (Mischel 1971, p. 326) o el isomorfismo fuerte (Murray 1979. p. $\times)^{30}$.

Thomas Kuhn popularizó, entre historiadores y filósofos de la ciencia (Kuhn 1972, p. 21), la tesis de que «la ontogenia cognitiva recapitula la filogenia científica». A la inversa, el historiador de la ciencia, Alexandre Koyré, dice que fue la física de Aristóteles la que le enseñó a comprender a los niños de Piaget. El filósofo Philip Kitcher afirmó recientemente (1988) que Ios psicólogos evolutivos pueden profundizar en los avances lingiísticos de los niños estudiando los cambios ocurridos en la historia de la ciencia; y que los historiadores y filósofos de la ciencia pueden aprender de los resultados experimentales y los análisis de los psicólogos infantiles. Kitchner (1985) proporciona una amplia bibliografía de las publicaciones filosóficas sobre Piaget.

Nussbaum (1983) hizo un primer artículo en relación con las publicaciones sobre cognición individual y cultural, o teoría diel desarrollo, en la enseñanza de las ciencias, titulado «Classroom Conceptual Change: the Lesson to be Learned from the History of Science». Carey ha advertido acertadamente que el éxito en captar la complejidad del cambio conceptual en los estudiantes de ciencias urequerirá la colaboración de científicos cognitivos y profesores de ciencias, quienes conjuntamente deben conocer la ciencia proporcinada por los historiadores y los fílósofos de la ciencia» (1986, p. 1125). Duschi, Hamilton y Grandy (1990) han publicado un amplio y extenso artículo al respecto.

El trabajo de Piaget conduce a una evidente área de investigación: ¿las concepciones intuitivas, inmediatas, "concretas" de los niños, reflejan los estadios primeros en el desarrollo del conocimiento científicoen los diferentes dominios? A un cierto nivel de simplificación, la respuesta es sí. Los niños parecen tener un conocimiento preinstructivo, o ideas previas, que son paralelas a las primeras nociones científicas o precientíficas. Esto ha sido ampliamente demostrado en el campo de la mecánica. McCloskey (1983), DiSessa (1982), Clement (1983), Champage (1980), Whitaker (1983), McDermott (1984) y Robin y Ohlsson (1989) son sólo algunos de los que sugieren que las ideas previas sobre fuerza y movimiento reflejan los fundamentos de Ia dinámica aristotélica. Bartov ha mostrado que las concepciones intuitivas de los procesos biológicos son altamente teleologicas (Bartov 1978). Otros apuntan a una explicación de tipo lamarckiano de la herencia en los niños (Brumby 1979). Furió, Pérez y Harris (1987) efectuaron un estudio sobre las creencias de los adolescentes en química, entre estudiantes que habían realizado cinco años de química en el instituto. Una proporción significativa continuaba apegada a la creencia de tipo aristotélico de que los gases no tienen masa, a pesar de las repetidas enseñanzas de la teoría atómica de los gases.

Este último asunto, la permanencia de creencias intuitivas, o ideas previas, a pesar de la instrucción científica -la demostración de McCloskey de que el $80 \%$ de los estudiantes universitarios de física continúan creyendo en el impetus es representativa de otros muchos estudios-, ha generado, por el contrario, intercambios beneficiosos, del tipo propuesto por Carey, entre profesores, psicólogos, filósofos e historiadores.

\section{IDEALIZACIÓN EN LA CIENCIA}

Existe, claramente, algo de importancia fundamental para entender el proceso cognitivo en esta resistencia de las creencias a la instrucción. Un aspecto filosófico es la naturaleza de la física clásica (newtoniana) y su relación con el sentido común y la observación. Con demasiada frecuencia, la ruptura epistemológica con el sentido común y nuestro mundo cotidiano, implicada en la ciencia, no ha sido reconocida, o ha sido descartada, en la instrucción científica y, de aquí, la aparente incapacidad de la instrucción para instruir se ha convertido en un enigma. Esta subestimación de la ruptura epistemológica con el mundo cotidiano, exigida por la mecánica clásica, es particularmente dominante entre aquellos profesores (la mayoría) que sostienen teoŕas empiristas de la ciencia. En cierta manera, esto es semejante al fracaso de los «paladines» del empirismo anteriores a Newton para entender correctamente la verdadera naturaleza de la revolución cientifica que ocurría en torno a cllos: una revolución que dependía más de la idealización, del análisis matemático y del experimento teóricamente apoyado que de la paciente observacion, tan recomendada por los filosofos empiristas y los autores de capítulos introductorios a los textos de ciencias (Mittelstrass 1972).

Duhem, a finales del siglo pasado, previno contra la fundamentación de la instrucción científica en el sentido común. Advirtió:

«Ahora bien, ¿está perfectamente claro, a la luz del sentido común, que un cuerpo en ausencia de cualquier fuerza actuando sobre él, se mueve perfectamente en línea recta con velocidad constante o que un cuerpo sujeto a un peso constante acelera constantemente la velocidad de su caf́da? Por el contrario, tales opiniones están enormemente Iejos del conocimiento de sentido común; para que aquéllos surgieran han sido necesarios los esfuerzos acumulados de todos Ios genios que a to largo de dos mil años han tratado de dinámica» (Duhem 1954 , p. 263).

Sesenta años más tarde, los principios pedagógicos suscitados por esta diyuntiva entre ciencia y sentido común, 
todavía ocupan la atención de los pedagogos con algún conocimiento de la historia y la filosofía de la ciencia. Champagne y otros expresaron el asunto en los siguientes términos: "lo más arduo del aprendizaje de la mecánica está en el esfuerzo requerido cuando los estudiantes cambian su pensameinto de un paradigma a otro. Los cambios de paradigma no se consiguen fácilmente, ni en la empresa científica ni en la mente de los estudiantes» (Champagne y otros 1980 , p. 1077). Pero, incluso donde se reconoce el cambio de paradigma, el efecto sobre los estudiantes para aprender y aceptar un nuevo paradigma, tan discordante con el sentido común y la observación, puede incluso subestimarse. El «cambio de paradigma» desde la mecánica medieval a la clásica, desde el conocimiento cotidiano al científico, no es tan sólo una cuestión de conseguir que los estudiantes vean las cosas de una manera diferente. Éste es todavía el modo aristotélico-empirista de tratar la cuestión: ver no es tan importante como esta tradición mantiene.

El entusiasmo de muchos filósofos y pedagogos por el problema de Kuhn del pato y el conejo, el problema de Hanson de la señora mayor y la joven, las figuras ambiguas y escondidas, los cambios en la «gestalt» (figura) y otras formas asociadas para representar la importancia de la teoría sobre la observación, o de la mente sobre la percepción, es comprensible, pero tiene una aplicación limitada para comprender la revolución científica. Simplemente la observación no jugó el papel que la mayor parte de estas representaciones sugiere. Decir que la teoría depende de la observación es interesante y puede servir para propósitos pedagógicos, pero no arroja luz sobre la revolución científica o la ciencia moderna. No es cl ver las cosas de forma diferente, sino el construir los objetos ideados, representarlos y manipularlos matemáticamente lo que diferencia la «nueva ciencia de una materia más antigua», usando las palabras de Galileo en la introducción a su Two New Sciences. Gatileo no «vio» las bolas sobre los planos inclinados como círculos incoloros sobre una tangente, él las vio simplemente como cualquier otro, pero las describió de una forma diferente y utilizó sus descripciones matemáticas en un nuevo aparato teórico; de forma similar Newion no «vio» péndulos como masas puntuales al fínal de una cuerda sin peso; él también los vio como cualquier otro, pero los describió de forma diferente y, una vez más, usó estas nuevas descripciones como ingredientes de un nuevo aparato teórico. Éstas son habilidades observacionales a desarrollar en la educación científica, pero no tienen la significación que frecuentemente se les atribuye (Norris 1985)

Un ejemplo muy simple ilustra la magnitud de la tarea. Todos los estudiantes de física aprenden de memoria la ley del isocronismo del péndulo: que el período de cada oscilación es el mismo de una oscilación a otra y que el período es independiente de la amplitud y de la masa del péndulo. En el texto de física del PSSC, el estudiante no pasa de la página cuatro cuando ya aprende esta ley y el supuesto descubrimiento por Galileo, mirando a un candelabro que se balanceaba y cronometrando su periodicidad. Sin embargo, está claro, que cualquier péndulo, de cualquier longitud y de cualquier masa, si se le hace oscilar, pronto se parará. Esto es una demostración conclusiva de que los péndulos no son isócronos; el período de la última oscilación no es el mismo que el de la primera. Un péndulo autéticamente isócrono oscilaría incesantemente, ¿Cómo reconciliamos la ley con la observación? La forma corriente de hacerlo es decir: «olvida lo que ves, aprende la ley». Una manera más sofisticada es decir: "la ciencia no trata realmente de esa clase de pénitulos que estás usando, trata de péndulos ideales en lo: que no hay fricción ni presión del aire, en los que la cuirda no tiene peso, etc.». Esto satisface al profesor conisedor de la historia y filosofía de la ciencia, pero ¿salisface al estudiante?

Scheker $(19 £ 8)$ se ha enfrentado a estas cuestiones de una forma in:eresante. Reconoce que el «principal progreso en la física de Galileo y Newton es liberar el pensamiento de las limitaciones de la experiencia directa, sensorial \{...] Los fenómenos directamente perceptibles y medibles son representaciones imperfectas del verdadero orden [que e: ] sólo accesible por medio de la idealización» (p. 217). Scheker pidió a 254 estudiantes de secundaria que comentasen la siguiente afirmación:

«En las lecciones de física hay con frecuencia presunciones o experimentos mentales que obviamente no pueden realizarse con experimentos reales, tales como excluir completamerte la resistencia del aire y otros efectos de fricción, o asumir un movimiento lineal que dure indefinidamente».

A los estudiantes se les preguntó si el método era útil o no. Un $11 \%$ dijo que no era útil:» ¿Por qué debo yo considerar algo que no existe?». Un extenso grupo, de hasta el $50 \%$ dijo que era útil, pero sólo para la física, porque la física no trata de la realidad: "No necesito referir todo a la realidad. Simplemente me interesa la física». Sólo aproximadamente el $25 \%$ tuvo alguna idea relativa al mistodo de idealización en ciencias.

La historia del estudio del movimiento del péndulo (Matthews 1987, 1990) puede arrojar mucha luz filosófica sobre tenas pedagógicos, particularmente el descubrimiento parádojico de que los estudiantes no creen que la física trate de la realidad. Galileo consideró su descubrimiento de la isocronía como esencial para el conjunto de su nueva física. Sin embargo, sus «descubrimientos» fueron vigorosamente refutados por Guilobaldo del Monte, que era el protector del propio Galileo y una persona descrita por Stillman Drake como «el más grande experto en mecánica del siglo xvi». La discusión entre ellos encubre la división epistemológica entre la antigua ciencia empírica aristotélica y la nueva ciencia idealista, matemática y experimentalista de la revolución científica. Del Monte le insistió a Galileo que los péndulos que usaba no eran isócronos: los de corcho y latón no tenían el mismo peníodo, los largos y los cortos no tenían el mismo período, todos los péndulos se paraban después de dos o tres docenas de oscilaciones. Galileo replicó que estos res altados se referían sólo a péndulos reales y que, si se estudiasen péndulos ideales (donde la friccion, la resistencia del aire y el peso de la cuerda se eliminan), entonces ciertamente se encontraría que eran isócronos. Galileo llegó a esta ley de isocronía por cálculo matemático (específicamente geométrico). Del Monte afirmó 
que las matemáticas estaban muy bien, pero que no cran física; la física debía ser sobre el mundo real, no sobre un mundo ideal. No sólo del Monte sino Huygens y muchos otros no creycron las afirmaciones de Galileo sobre el movimiento isócrono del péndulo; Huygens dijo que Galileo «debe haber inventado los experimentos más que haberlos realizado». No sorprende que cuando los profesores confían en un método simple, sin guía de descubrimiento, los estudiantes encuentren el resultado de del Monte, no el de Galileo. Un poco de historia de la ciencia puede preparar a los profesores para este resultado. Un poco de filosofía de la ciencia puede ayudar a los profesores a interpretar los resultados para los estudiantes.

Hay una diferencia entre los objetos reales del mundo y los objetus teóricos de la ciencia. Confundir el primero con el segundo es confundir la ciencia aristotélica con la newtoniana. Di Sessa señaló, sobre los fallos del aprendizaje a partir de descubrimientos estándard, que «parece que ruy pocos estudiantes, si hay alguno, habían adquiridc más sentido newtoniano que tratando con el mundo cotidiano [...] los experimentos mentales puede que sean más útiles que andar enredando» $(1982$, p. 62). Esperar cue los estudiantes aprendan algo newtoniano juguetearido con los objetos es subestimar la revolución epistemológica inaugurada por Galileo y Newton. Y es también subestimar los problemas pedagógicos para comprender la visión científica clásica del mundo. «Itlgar con»o «mirar prolongadamente a», objetos materiales reales no generárá los puntos másicos, los cuerpos inerciales, las definiciones de fuerza, la geometría y el cálculo que son parte esencial de los objetos teóricos de la mecánica. Los primeros entran en la ciencia cuando se los representa en términos de los segundos. Una vez así preparados, y dentro del sistema, se puede operar sobre ellos con el aparato conceptual de la ciencia.

Algunos temas análogos se discuten en cl detallado trabajo histórico-psicológico de Steinberg, Brown y Clement (1990) sobre «The Conceptual Difficulties Impeding, Isaac Newton and Contemporary Physics Students". El relativamente olvidado asunto de los experimentos tnentales en la cnseñanza de las ciencias se comenta en Helm, Gilbert y Watts (1985), Stinner (1990), Winchester (1990) y Matthews (1989a).

Los principios filosóficos asociados con la idealización y la abstracción en la ciencia tienen, desde luego, implicaciones para las publicaciones sobre enseñanza de las ciencias relativas a los abundantes crrores conceptuales (Helm 1980), o concepciones alternativas (Driver and Easley 1978), o ideas previas. Volviendo a del Monte: ćl no tenía errores conceptuales sobre el movimiento del péndulo, sólo los tenía sobre la nueva visión de la ciencia del movimiento del péndulo. Se dice comúnmentc que los aristotélicos tenían errores conceptuales sobrc el mundo real; sería más acertado decir que tenían errotes conceptuales sobre el mundo inventado y construido por Galileo y Newton, es decir, sobre los objetos téóricos de la nueva ciencia, no sobre los objetos materiales que trata. Los principios filosóficos tienen también implicaciones para la exposición del conflicto cognitivo piagetiano del cambio conceptual: está claro que la experiencia no va a proporcionar el conflicto cognitivo que es supuestamente el motor del cambio conceptual. Repitiendo lo que se ha dicho: la experiencia es muy aristotélica; tanto, que un destacado historiador de la revolución científica dijo que «la observación y la experiencia $[\ldots]$ tenían un papel muy pequeño en la edificación de la ciencia moderna; se podría incluso decir que constituyen los principales obstáculos que tal edificación encuentra en su camino» (Koyré 1968, p. 90. $\mathrm{El}$ reconocimiento de la idealización científica provoca muchas preguntas en este terreno: ¿Qué es un error conceptual? ¿Es el mundo real la piedra de toque contra la que juzgamos nuestras concepciones, o la piedra de toque es realmente otra conceptualización del mundo? El tema de la idealización está siendo últimamente considerado en las publicaciones sobre HFC y la enseñanza de las ciencias.

Floden y otros (1987) han discutido los problemas pedagógicos ocasionados por la tuptura entre la enscĩanza de las ciencias y la experiencia cotidiana. Garrison y Bentley (1990) desarrolian el tema en una discusión sobre la teoría del cambio conceptual del influyente Posner y otros (1982). Ginev proporciona una explicación muy sofisticada de los currículos de ciencias construidos sobre el reconocimiento de que «el proceso mismo de idealización es considerado como la diferencia especifica epistemológica de la ciencia» $(1990$, p. 65).

La historia y la filosofia pueden hacer las idealizaciones de la ciencia más humanas, comprensibles, y explicarlas como útiles con derecho propio para ser apreciadas. Esto es importante para los estudiantes a quienes se introduce en el «mundo de la ciencia». La incapacidad para apreciar qué es la idealización y qué no es ha sido la base de una gran cantidad de críticas anticientíficas. Fue, por supuesto, la idealización newtoniana aquélla contra la que se đirigió la reacción de los románticos. Para ellos (Keats, Goethe, etc.), el rico mundo de la experiencia vivida no fue capturado por las incoloras masas puntuales de Newton. En el sigloxx, Marcuse, Husserl, Tillich y otros han repetido versiones de estas objecciones. El destacado pedagogo-científico alemán, Martin Wagenschein (1962) ha escrito sobre esta dicotomía en el meollo de la enseñanza de las ciencias. El punto que debe enfatizarse aquí es que Ios objetos teóricos de la ciencia no están propuestos como explicaciones de los objetos materiales del mundo, al menos no en el sentido de imágenes suyas.

Un profesor de ciencias instruido en bistoria y filosofía puede ayudar a los estudiantes a entender cómo la ciencia capta, y no capta, el mundo real, subjetivo, vivo. Es más común dejar al estudiante con la desgraciada alternativa de rechazar su propio mundo como una fantasía. Siguiendo a Macb, una vez más, el mundo vivo de los fenómenos es vital para la instrucción científica, que es donde la curiosidad y la preocupación comienzan, pero no debe confundirse con un mundo inercial o el mundo de los gases ideales. Algunos de estos asuntos sc comentan en Eger (1972) y Passmore (1978). 


\section{FILOSOFÍA DE LA CIENCIA Y ENSEÑANZA DE LAS CIENCIAS}

Hace diez años, Ennis comenzó una revisión global de la literatura americana sobre filosofia de la ciencia y la enseñanza de las ciencias (el único artículo sobre enseñanza de las ciencias que aparece en una revista de fílosofía de la ciencia en décadas), con esta melancólica observación «Con algunas excepciones, los filósofos de la ciencia no han mostrado explícitamente mucho interés en los problemas de la enseñanza de las ciencias» (Ennis 1979 , p. 138). Se olvidaba de Inglaterra y de la significativa contribución de Whitehead (Birch 1988). Si Ennis podía entonces contar el número de tales filósofos con los dedos de las manos -Dewey, Scheffler, Martin, Margenau, Nagel-, ahora pueden ser contados, por los menos, con dos manos. Algunos nombres que se podrían añadir son: Siegel, Harre, BuchdahI, Ruse y Pitt. Entre los pedagogos sobre la ciencia, Richard Dushen Ios Estados Unidos, Derek Hodson, en la comunidad británica, y Walter Jung, en la comunidad europea, han abogado recientemente por una mayor cooperación con los fílósofos de la ciencia.

El filósofo que más contribuyó a abrir el diálogo entre la historia y la filosofía de la ciencia y la enseñanza de las ciencias fue Michael Martin en su famoso Jibro Concepts of Science Education (1972). Tanto Ennis como Martin eran filósofos analíticos. En cinco capítulos que tratan de investigación, explicación, definición, observación y objetivos, Martin proporciona una amplia muestra de la utilidad de la filosofía para la mejora de la formación, los textos y las propuestas de metas y objetivos en un curso de ciencias.

Dos ejemplos dan una idea de la contribución de Martin. Apunta que la explicación es esencial para la instrucción en ciencias y que el concepto aparece reiterativamente en distintos textos, mencionando específicamente el Biological Science: An Inquiry into Life, de BSSC, y el Investigating the Earth, de ESCP. Sin embargo, en ninguno de estos textos se intenta aclarar qué es una explicación científica y cómo las buenas se pueden diferenciar de las malas. Dice que una elaboración elemental del modelo siguiendo una ley, y los modelos estadísticos-probabilísticos, no harán ningún daño y, por el contrario, sí que probablemente harían mucho bien. En el último capítulo reproduce una declaración de principios para la enseñanza de las ciencias, del entonces importante Education and the Spirit of Science, del US Educational Policies Commision (1966). En su tercera meta sobre enseñanza de las ciencias, dice:

«El hambre de saber es la motivación para aprender:datos y generalización son las formas que toma el conocimiento. Las generalizaciones son inducidas de bits aislados de información, recogidos a través de la observación dirigida tan acertadamente como las circunstancias lo permitan.»

Martin apunta, en contradicción con esto, que algunas teorias científicas no son generalizaciones y que la mayor parte de Ias hipotesis científicas no son generadas por inducción y, aún más, que la observación requiere teoría.
Este último asunto, el desconocimiento de la observación a la teoría, y sus implicaciones, ilustra bien la lamentable separación entre los filósofos de la ciencia profesionales, los educadores de ciencias y los órganos consultivos gubernamentales. El informe The Spirit of Science fue publicado en 1966. El inductivismo estaba en ese momento en plena retirada de la filosofía de la ciencia: Paterns of Scientific Discovery, de Hanson, había aparecido en 1958; Logic of Scientifis Discovery, de Popper, había sido traducido en 1959; Light and Understanding, de Toulmin, aparecio en 1961; Structure of Scientific Revolutions, de Kuhn, apareció en 1962. Había cantic ad de material a mano, por no mencionar el material del otro lado del Atlántico (Bachelard) y material remontándonos en el tiempo (Collingwood, Fleck), el cual podía haber documentado las deliberaciones de una agencia de tan alto rango en el gobierno de los Estados Unidos al emitir un importante informe sobre enseñanza de las ciencias. En cambio, el material fue ignorado y se promulgaron controvertidos eslógans, sin que hubiera: s sido discutidos, ni mucho menos que cupiera la posibilidad de que fueran falsos.

No eran sólo los informes gubernamentales los que padecían la ignorancia del desarrollo de la filosofía de la ciencia. Rot ert Gagné, uno de los más influyentes teóricos sobre el aprendizaje entre 1960 y 1980 y una figura prominente :ras los curriculos de investigación-aprendizaje de cien aias, prestó en 1963 su considerable prestigio a la debatible visión de la investigación científica como investigación, donde:

«un conjun:o de actividades que comienzan con un cuidadoso conjunto de observaciones sistemáticas continúa diseña ado las medidas pertinentes, distingue claramente entre lo que se observa y lo que se infiere [...] y saca conclu siones razonables (Gagné 1963, tomado de Hodson, próxima publicación).

Numerosos proyectos curriculares de NSF a principios de los años 60 y el esquema del Nuffield de 1960 en Inglaterra a Jolecieron, igualmente, de la ignoracia del desarrollo de la filosofía de la ciencia, propagando una aproximación a la ciencia por el descubrimiento como réplica a los métodos supuestamente inductivos de la ciencia. El eslógan de Bruner - "produce científicos haciendo que los estudiantes sean científicos»- sería admirable si los profesores y los diseñadores curriculares tuvieran unsı idea razonable de lo que es ser un científico. La mayor parte aceptó la mitología inductivista de los libros de texto. Stevens (1978), Forge (1979) y Dushl (1985) han iscrito sobre los supuestos filosóficos de los currículos deI NSF y Nuffield.

Si documeritos normativos, destacados teóricos de la enseñanza, currículos y libros de textos aceptan y, sin crítica alguna, promulgan ciertas posiciones filosóficas, no es sorprendente que los profesores de ciencias hagan lo mismo e 1 el aula. La teoría de un profesor sobre la naturaleza cle la ciencia -la epistemología del profesorpuede ser comunicada explícita o implícitamente. Esta epistemoloyía afecta a la actuación de los profesores en el aula (Robinson 1969). Cómo se forma esta epistemología, qué afectos tiene sobre la práctica educativa y 
cómo contribuye a la imagen que el estudiante se forma de lat ciencia han sido los objetivos de muchos estudios recientes: Abell (1989), Rowell y Cawthron (1982), Jacoby y Spargo 81989), Lederman y Zeidler (1987), Koulaidis y Ogborn (1989). Esta investigación es más importante cuando se ponen en marcha esquemas tales como cl currículo nacional británico, el proyecto 2061 y otros en tos que tópicos como sla naturaleza de la ciencia» son parte del currículo.

La epistemología de un profesor se crea informalmente; está recogida en la descripcción de Kuhn sobre la evolución de los libros de texto. Consiste en los prejuicios en boga que han sidoescasamente afectados por la información histórica o el análisis fílosófico. Sólo en dos de las 55 instituciones que forman profesores de ciencias en Australia, hay un curso obligatorio en HFC. De los quince principales centros que forman profesores de ciencias en Estados Unidos, sólo la mitad exige un curso en filosofía de la ciencia (Loving 1992); la proporción en los restan. tes cientos de centros es seguramente menor. La situación en Gran Bretaña no es más alentadora.

\section{HISTORIA Y FILOSOFÍA DE LA CIENCIA Y FORMACIÓN DEL PROFESORADO}

Mucha gente ha defendido que HFC deberían estar inciuidas en la formación de los profesores de ciencias; el británico Thomson Report ya decia, en 1918, que «algún conocimiento de la historia y la filosofía de la ciencia debería formar parte del bagaje intelectual de todos los profesores de ciencias en la enseñanza secundarias (p. 3). Un argumento a favor de HFC es que produce una enseñanza mejor (más coherente, estimulante, crítica, humana, etc.). Este argumento utilitario no es el único; otro puedes ser que los profesores adquieran un conocimiento crítico (conocimiento significativo histórico y filosófico; de su asignatura con independencia de que este conocimiento sea utilizado pedagógicamente: hay algo más, para el profesor, que hacer frente a la clase.

Michael Polanyi resaltó como obvio que HFC deberían ser, al metıos, parte de la enseñanza de la ciencia, como la crítica literaria y musical lo son de la enseñanza literaria y musical (Harre 1983, p. 141). Sería extraño pensar en un buen profesor de literatura que no tuviera conocimiento de elementos de crítica literaria: del tradicional debate sobre qué iđentifica a ta buena literatura, cómo la literatura se relaciona con los intereses sociales, la historia de las formas literarias, etc. Así, también sería igualmente extraño pensar en un buen profesor de ciencias que no tenga un conocimiento razonablemente elaborado de los términos de su propia disciplina -causa, ley, explicación, modelo, teoría, hecho- o un conocimiento de los objetivos, frecuentemente conflictivos, de su propia disciplina -describir, controlar, comprender-o un conocimiento de la dimensión cultural e histórica de su propia disciplina. Israel Scheffler comentaba estas cuestiones en un trabajo de 1970 , durante mucho tiempo olvidado. Issto parte de la diferencia entre ser educado en ciencias y simplemente ser formado en ciencias: los profesores deberían ser educados. HFC contribuyen claramente a esta rica comprensión de la ciencia.

Éste es un punto de contacto con la voluminosa literatura sobre «alfabetización» científica: si estar alfabetizado es comprender con cierta profundidad las palabras y conceptos de un discurso, entonces la historia y filosofía de la ciencia contribuyen claramente a una «alfabetización» científica más profunda y crítica (Miller 1983).

La defensa de la importancia de la HFC para los profesores de ciencias no es nueva. Las páginas iniciales de un texto de 1929, para profesores de ciencias, describe al profesor con éxito como aquél que: «sabe su propia asignatura [...] está ampliamente instruido en otras ramas de la ciencia [...] sabe cómo enseñar [...] es capaz de expresarse con lucidez [...] es hábil manipulando [...] tiene recursos tanto para las demostraciones teóricas como para el laboratorio $[\ldots]$ es un lógico [...] es algo filósofo [...] es tan buen historiador que puede sentarse con un grupo de alumnos y hablarles de las ecuaciones, la vida y los trabajos personales de genios tales como Galileo, Newton, Faraday y Darwin (citado en Sherratt 1983, p. 418).

Este ideal tiene relevancia actualmente. Como se ha mencionado antes, los nuevos currículos que se están desarrollando e implantando en Gran Bretaña, Estados Unidos, Dinamarca y Canadá requerirán todas estas cualidades en un profesor, si esos currículos se han de enseñar con éxito. Episodios de historia de la ciencia y cuestiones sobre la naturaleza (filosofía) de la ciencia son parte de esos currículos. Aún más, en Gran Bretaña, Estados Unidos, Australia y muchos otros lugares, se están haciendo esfuerzos para identificar a los profesores de ciencias destacados y para evaluarlos. Esto requiere que se definan las cualidades de un buen profesor de ciencias. Cada vez, con mayor frecuenciá, se requiere cierta competencia o familiaridad con temas de HFC.

En Estados Unidos, el National Teacher Assessment Project, ubicada en Stanford, con fondos de la Carnegie, dirigida por Lee Shulman, es el más avanzado programa de evaluación de profesores. Es intelectualista en sus criterios sobre la competencia de los profesores y rechaza el conductismo -dirigismo-medidas de la competencia de los profesores que desde hace tiempo son reliquias en la práctica evaluadora. Shulman pregunta sobre el «paradigma desaparecido» -el dominio de la asignatura- y la habilidad para hacerlo inteligible a los estudiuntes, habilidades que requieren la más amplia visión proporcionada por HFC. En una de sus publicaciones, Shulman ha dicho:

«Pensar con propiedad sobre el conocimiento del contenido requiere ir más allá de los hechos o conceptos de un campo. Requiere comprender la estructura de la asignatura [...] Los profesores no sólo deben ser capaces de definir a los estudiantes las verdades aceptadas on un campo. Deben también ser capaces de explicar por qué una cierta proposición se estima justificada, por qué vale la pena conocerla y cómo se relaciona con otras propo- 
siciones, tanto dentro de la disciplina como fuera de ella, tanto en la teoría como en la práctica (Shulman 1986 , p. 9).

Explicar por qué cierta proposición se estima justificada - la ley de la inercia, el principio de conservación de la energía, la teoría de la evolución, la teoría del desplazamiento de los continentes, la descripción de la estructura atómica, etc. - requiere saber algo sobre cómo las pruebas se relacionan con la apreciación teórica; ésa es la función normal de la epistemología. Las ideas de Shulman están contenidas en las líneas directrices del National Board for Professional Teaching Standars Assessment: What Teachers Should Know and Be Able to Do (1989).

Un paquete de evaluación para profesores de biología, que se ha desarrollado como parte del proyecto Carnegie, intenta evaluar lo que los profesores han captado de la naturaleza de la ciencia, sus procesos y factores determinantes. Usando sus palabras: «Los profesores tienen una concepción rica de la empresa científica como una interacción de hechos, leyes y teorías de un campo, dominando las habilidades para construir tal conocimiento y reconociendo que este conocimiento está influenciado por la sociedad humana y ha tenido influencia en ella» (Collins 1989, p. 64).

A medida que HFC se convierten en un componente reconocido de la educacción del profesor, es oportuno preguntarse qué clase de cursos de HFC son adecuados. La literatura reciente contiene información sobre un número considerable de tales cursos y reflexiones sobre su adecuación. Existe consenso en que para que dichos cursos se consideren relevantes para el futuro profesor deberían ser cursos aplicados o prácticos. Enviar estudiantes de pedagogía a un departamento de filosofía para hacer HFC no es el modo más satisfactorio de proceder. Utilizando las palabras de Bevilacqua que está promoviendo $\mathrm{HFC}$ entre los profesores de Italia, tales cursos se convierten en otro «ladriflo en la pared» u otra tarea a completar antes de lanzarse a la enseñanza. Los cursos de HFC deberían comenzar con problemas que los profesores vean como pertinentes en sus enseñanzas o desarrollo profesional. Algunos cursos están descritos en Jhonson y Stewart (1990), Eger (1987), Bybee (1990), Bakker y Clark (1989) y Ruse (1990).

Mi propio curso (Mathews 1990 b), que se ha impartido con algún éxito durante algunos años, se basa en selecciones de los escritos de Galileo, Boyle, Newton, Huygens, Darwin y otros. He descubierto, sin que me sorprenda, que los profesores aprecian la oportunidad de leer algo de esos trabajos. De cientos de licenciados en biología, he encontrado sólo un puñado que haya leído algún escrito de Darwin; de cientos de licenciados en física, no he encontrado ninguna que haya leído algo de Galileo o Newton. Como un profesor dijo: «los profesores estamos hambrientos de estos conocimientos». Los conceptos filosóficos -realismo, instrumentalismo, autoridad, reduccionismo, causalidad, explicación, idealización, etc.- se tratan y desarrollan a medida que surgen en el texto. La mayor parte de los textos utilizados han sido publicados en Matthews (1989b).

\section{ALGUNAs CUESTIONES DE INTERÉS ACTUAL}

Ennis, en 19\%9, relaciona seis áreas de interés para los profesores de ciencias que se beneficiarían con las consideraciones filosóficas. Éstas son: el método científico, los criterios para un pensamiento crítico sobre enunciados empíricos, la estructura de las disciplinas científicas, la explicación, la valoración de las opiniones de los cientíiicos y la realización de exámenes. Diez años más tarłe, aún es procedente sacar esta lista. Los temas que yo propondría son: feminismo, constructivismo, ética, metafísica, idealización y racionalización. De una u otra forma, estos temas han estado en el fondo del debate sobre la educación en ciencias. Esto no quiere decir que asuntos más comunes no debieran ser discutidos; una investigación en Australia demostró que, aproximadamente un tercio de la clase de química de primero en una universidad, no sabía que «afirmar el consecuente; era un razonamiento no válido; creían que si A implica B, y B es cierto, entonces A es también verdad. Esto sugiere que tratar de las habilidades de lógica simple y de razonamiento no está fuera de lugar, aunque no se puedan tratar asuntos más complejos.

1) El feminismo ha suscitado grandes desafíos, tanto a los supuestos sobre la enseñanza de las ciencias como a los de la filosofía de la ciencia. Es notorio que las mujeres no :iguen los estudios de ciencias. Existen montañas de titeratura sobre el tema (The International Journal of Sitience Education, especial 1987). Buena parte de su contenido es empírico, tratando de los impedimentos al progreso de las mujeres y su interés por la ciencia. Adenás, existe una reivindicación filosófica que exige respuesta. Bleir (1984), Harding (1986), Keller (1985) y Martin (1989) han discutido el caso de la parcialidad mtasculina en la epistemología misma de la ciencia occidental. Los pedagogos sobre ciencias han empezado a prestar atención a esta crítica. Martin ha dicho que «traer la crítica basada en el género a la educación cicntífica es incorporar la enseñanza acerca de la ciencia en la enseñanza de las ciencias» (Martin 1989 , p. 251). Se sabe que las ideologías de clase, raza y religión han afectado el desartollo de la ciencia: la genética de Lysenko, los hematólogos nazis buscando un tipo de sangre judía y aspectos de la ciencia medieval proporcionan ejemplos de cada una de ellas. Así pues, $a$ priori, es posible que una ideología machista pueda afectar a la ci incia, incluso a su epistemología. Harding proporciona una visión de un nuevo tipo de conocimiento promovido por el feminismo, éste busca una unidad de conocimientc combinando lo moral y lo político con el conocimiento empírico. Y busca unificar el conocimiento desde y por el corazón con lo que se consigue por y mediante el cerebro y la mano. Contempla lá investigación comprendiendo no sólo la observación mecánica y otras de la raturaleza, sino también la intervención de hechos políticos y morales «sin los que los secretos de la naturaleza nc pueden ser desvelados» (Harding 1986, p. 24).

Esta es una demanda epistemológica y necesita ser defendida colherentemente. Requiere prestar atención a la historia y la filosofía de la ciencia. 
2) El constructivismo puese decirse que es la epistemología dominante entre los pedagogos de ciencias. Está articulado en los trabajos de von Glasersfeld (1989), Novak (1987), Driver (1985) y muchos otros. Un con mentario reciente ha identificado las siguientes escuelas o variantes de constructivismo: contextual, dialéctico, empírico, procesador de información, metodológico, moderado, piagetiano, postepistemológico, pragmáti$c o$, radical, realista, social y sociohistórico; a lo que se puede añadir, humanístico (Cheung y Taylor 1991). El constructivismoes para los piagetianos, cognitivo, siguiendo la teoría tradicional, opuesto al conductivismo de tipo skineriano o gagneiano, que durante tanto tiempo han dominado en la educación científica. Como en la mayor parte de esta tradición, su teoría de la mente es fundamentalmente kantiana. La ontología del constructivismo varía desdc el idealismo radical (particularmente on algunos de los escritos de von Glasersfeld) hasta ia teoría de los tres mundos de Popper. Su práctica pedagógica es antididáctica y centrada en el estudiante, haciendo énfasis en el compromiso del estudiante con la identificación de problemas, desarrollo de hipótesis, comprobación y discusión.

Hay una cantidad de cuestiones filosóficas en la teoría constructivista que merecen elaboración: ¿Qué valor se da a la dimensión social del conocimiento? ¿Cuáles son los criterios para la adecuación de las concepciones del estudiantc. ¿Se juzgan frente a normas de la comunidad científici, o frente a comentarios de otros estudiantes, o frente a las concepciones previas del individuo? ¿Existe confusión entre práctica pedagógica exitosa y exigencias epistemológicas? Driver, en una de sus publicaciones, reconoce una tensión esencial en la práctica constructivista: Ia que hay entre hacer que los estudiantes construyan y den significado a sus propias versiones sobre algo, y hacerlos participar en una comunidad cientifica que tiene sus propias teorías y conocimientos (Driver y Oldham 1986). La primera no hace un juicio epistemológico, sólo se refiere a mecanismos psicológicos; la segunda hace juicios epistemológicos. Los profesores que quieran que sus estudiantes entren y dominen esta esfera del conocimiento público necesitarán normalmente indicar que las explicaciones que los estudiantes pueden elaborar y con las que estarán contentos son, de hecho, inadecuadas. Strike (1992) y Gruender (1989) han planteado algunas de estas cuestiones; Suchting (1992) proporciona una crítica penetrante.

Un problema profundamente arraigado en muchos de los escritos de los constructivistas es que el constructivismo mantiene básicamente una concepción empirista del conocimiento, a pesar de su expresada antipatía por tales puntos de vista. Esto surge cuando los constructivistas dicen que sólo somos conscientes de las impresiones de nuestros sentidos, que no podemos tener acceso al mundo tal cual es, que el conocimiento es la correspondencia de la imagen mental o las ideas con la realidad, que es el sujeto individual el escenario de las pretensiones del conocimiento. Todas éstas son reivindicaciones empiristas estándard. Pero el conocimiento científico requiere idealizaciones, y éstas no pueden, por definición, reflejar $\mathrm{o}$ corresponderse con el mundo real. Por tanto, $o$ la correspondencia se ha de abandonar como criterio de conocimiento, o ninguna rama de la ciencia moderna puede ser aceptada como conocimiento. Lo primero parece más sensato. Pero si se cumple, entonces desaparece una gran parte del argumento constructivista sobre el relativismo.

3) Cada vez surgen más cucstiones éticas en casi todas las áreas del currículo de ciencias. El efecto invernadero, la contaminación, la extinción de las especies, la ingeniería genética, la tecnología militar y cl empleo de científicos en las industrias de defensa, el coste y la dirección de la investigación científica, la energf́a nuclear y la guerra nuclear y otros muchos asuntos son temas que los alumnos suscitan y que aparecen en los nuevos currículos de ciencias. Ahora se cuestiona el antaño inexcusable e irreflexivo uso de los animales para experimentos científicos y disecciones de laboratorio y en muchos lugares se limitan severamente. Un objetivo del programa de ciencias de Nueva Zelanda es el «cuidado de los animales» y el reconocimiento de sus derechos. A1 mismo tiempo que en filosofía, estas cuestiones se cstán tratando en curso de ética aplicada y de ética del medio ambiente. Hasta ahora, en parte por la influencia de la creencia en una ciencia libre de valores, estas cuestiones han sido ignoradas en la enscñanza de las ciencias. Ya no pueden ser ignoradas por más tiempo.

John Ziman y muchos otros han indicado currectamente que la enseñanza ortodoxa de las ciencias ha fomentado durante mucho tiempo el materialismo ingenuo, el positivismo primitivo y la tecnocracia autosuficiente ( $Z$ iman 1980). Estas posiciones (ideologías)están ahora cn retirada intelectual. Ahora debemos enfrentarnos con las consecuencias pedagógicas de la «muerte de la ciencia libre de valores». El proyecto PLON en Holanda, el SISCON en Gran Bretaña, varios proyectos canadienses (Aikenhead 1980), los cursos CTS y las proposiciones del proyecto 2061 en los Estados Unidos son las respuestas curriculares más obvias. Sc ha generado una literatura considerable sobre ćtica en la enseñanza de las ciencias (Mendelsohn 1976, Gosling y Musschenga 1985). Los profesores pueden beneficiarse considerando algunos argumentos adelantados por los filósofos que han tratado temas similares.

El reciente intercambio entre Eger, Hesse, Shimony y otros (Zygon $23(3), 1988)$ sobre "racionalidad en las ciencias y en la étican (reproducido en Matthews 1991) muestra lo que se puede conseguir con una colaboración de este tipo. Eger (1989) ha tocado también las cuestiones de los «intereses» de la ciencia, tomando temas que el trabajo de Habermans y la escuela de Frankfurt presentan para comprender el papel social de la ciencia y las estructuras fundamentales de la disciplina.

4) Los temas metafísicos emergen naturalmente de la materia de la propia ciencia: Einstein describió al científico como un filósofo en ropa de faena. Los temas de la ética en la ciencia suscitan cuestiones sobre nuestra responsabilidad con la naturaleza que, por su parte, es impulsada por una nueva concepción de la naturalcza misma. La visión mecánica, laplaciana, del mundo de la ciencia newtoniana está siendo retada por una nueva ontología de la naturaieza. Gotschl (1990) dice que «el 
amplio llamamiento a la responsabilidad se traduce en una revolución ética y antropológica, al lado de la revolución científica y técnica». Los profesores deberían tener alguna idea de estos asuntos: existe una gran cantidad de ontologías que compiten en el mercado por reemplazar la visión mecánica del mundo; algunas tienen mucho más sentido que otras. Un profesor de ciencias versado en $\mathrm{HFC}$ puede evaluarlos.

Los estudios históricos de la ciencia reflejan vívamente la independencia de la ciencia y la metafísica. La controversia gallileo-aristotélica sobre las causas finales, la controversia galileano-kepleriana sobre la teoría lunar de las mareas, la discusión cartesiano-newtoniana sobre la acción a distancia, la discusión newtontano-berkeliana sobre la existencia de un espacio y un tiempo absolutos, la discusión newtoniano-fresneliana sobre la teoría corpuscular de la luz, la discusión darwiniano-paleyana sobre destino y selección natural, la discusión MachBohr sobre la teoría atómica, la disputa Einstein - escuela de Copenhague sobre la interpretación "determinista» de la teoría de los cuantos, etc., todas sacan a colación temas metafísicos. La metafísica lo impregna todo en la ciencia. Peirce había dicho en sus Notas sobre Filosofía Cientifica: «Busca un hombre de ciencia que proponga mantenerse sin metafísica [...] y habrás encontrado uno cuyas doctrinas están totalmenete viciadas por la cruda y no valorada metafísica de la que están impregnadas.» Estas interconexiones se discuten en Wartofsky (1979), Gjertsen (1989) y Matthews (1989b).

Woolnough (1989) ha señalado una cuestión importante en la historia de la ciencia que normalmente se ignora en los programas de ciencias: el papel de las creencias religiosas en la motivación y conceptualizaciones de los grandes científicos. Los estudiantes aprenden con bastante frecuencia que Newton dijo, cuando escribió sus Principia, que la base de toda la ciencia moderna «tenía una visión de cómo podrían funcionar tales principios considerando a los hombres creyentes en una divinidad, y nada puede regocijarme más que encontralo útil para ese fin» (Thayer 1953. p. 46). Los estudiantes también aprenden con demasiada frecuencia que Boyle descubrió una ley importante y cuál es su fórmula; aprenden con menos frecuencia que dejó una cláusula en su testamento para que se hicieran una serie de lecturas públicas destinadas a «probar la religión cristiana contra infieles notorios", y que creía que su propia fílosofía mecánica era admirablemente adecuada para probar la existencia đe un «diseñador» del universo. A pesar del hecho de que la historia de la ciencia occidental es en gran parte una historia de los esfuerzos de la gente que se veía a sí misma haciendo un trabajo que proclamaba la majestad de Dios, no se oye hablar mucho de este tema en el tratamiento que habitualmente se hace, en el aula de ciencias, de estas figuras y sus descubrimientos. Existen interesantes historias psicológicas, culturales y filosóficas que pueden narrarse con gran provecho.

Si la ciencia se ha desarrollado como un diálogo con la metafísica (por no decir nada sobre intersecciones con los campos político, económico y social), enseñar la ciencia como un soliloquio en que la propia ciencia se habla simplemente a sí misma y crece única- mente con su autocrítica, entonces, es empobrecer su contenido.

El propio contenido de la ciencia también suscitará cuestiones nietafísicas. El biólogo Charles Birch enuncia esto en sı nivel más general: «cualquier profesor de ciencias transmite finalmente sus ideas en la respuesta a la pregunta: ¿de qué está hecha la naturaleza? [...] La respuesta dcminante hoy en día se da en términos del modelo mecanicista de la naturaleza» (Birch 1988, p. 33). Birch argumenta, en contra del mecanicismo, la visión cartesiana del mundo, en favor del proceso metafísico de Writehead. Siguiendo a este filósofo dice que «el papel de la educación es tratar con hechos e ideas, de tal forma quŁ nos quede una comprensión generalizada»; la metafísica figurará en esta comprensión. Thomas Settle maneja de una forma admirablemente no técnica la cuestión suscitada por Birch: "¿puede el fisicalismo ser evitado en la enseñanza de las ciencias en la escuela? (1990). Es cigno de mención que éste y otros trabajos (Stinner 1990) están resucitando las visiones filosóficas y pedagógicas de Alfred North Whitehead, que fueron publicadas for primera vez en su The Aims of Education (1923).

La consider:able literatura generada por el movimiento de «filosofía para los niños» sugiere que éstos son capaces de ieguir cuestiones elementales filosóficas y de estar intiresados en elias. (Lipman y Sharp 1978, Dawson-Galle 1990). La clase de ciencias proporciona grandes oportunidades para hacerlo.

5) La idealizıción es la condición sine qua non de la moderna ciencia mat emática. Sin embargo, es muy poco comprendida por los profesores; raramente se encuentra tratada en los textos sobre el método científico y ignorada frecuentemente por los filósofos que siguen las dicusiones sobre inducción, falsación y comprobación de las teorías, cormpletamente ajenos al hecho de que son las leyes y las tyorías idealizadas las que se discuten, y que la simple lógica es inadecuada para su evaluación. Por otra parte, nucha literatura relativa a la enseñanza de las ciencias sol.re adquisición de conceptos procede de una forma aristcitélica, en la que las idealizaciones se tratan como generalizaciones empíricas. Claramente, la adquisición de los conceptos de masas puntuales, superficies sin rozamie!lto, sistema inercial, colisión elástica, sólido rígido, etc. no se produce de forma aristotélica, no surge de mirar los cuerpos e inducir características comunes. La idealizasión galileano-newtoniana fue un Iogro conceptual morumental indiscutiblemente, algo que separa el pensamiento humano de toda percepción animal. Este logro debe hacerse llegar a los estudiantes, que no adquirirán las idealizaciones mirando la naturaleza. Destacados zilósofos de la ciencia han tratado ampliamente la lógica y filosofía de la idealización en las ciencias (Novak 1980, Krajewski 1982).

Debe recordarse la feliz expresión de Duhem: «la lógica de un tema no es necesariamente Ia lógica de su presentación». La historia alerta a los profesores sobre la necesidad de una aproximación fenomenológica a las idealizaciones: los estudiantes necesitan saber con qué se relacionan las idealizaciones. 
6) La racionalización es el tópico que une HFC con la enseñanza de las ciencias. En HFC la racionalidad del cambio científico teórico, es decir, de la historia de la ciencia, ha sido un asunto fuertemente controvertido. Los racionalistas despertaron de su sueño con la bomba de la Structure de Kuhn de 1962, en la que se decía que las transformaciones científicas dependen no tanto de la persuasión racional como de la psicología de la muchedumbre y la mortalidad de la gente mayor. Justo cuando los filósolos se reconciliaban con ello y con la extensión de la tesis de Feyerabend (ver las defensas del racionalismo en Shapere 1984 y Siegel 1987), la escuela de sociólogos de la ciencia de Edinburgo -David Bloor, Barry Barnes, Steven Shapin y Michael Mulkay- criticaba aún más el racionalismo, en su duro programa, donde tralan de dar cuenta de todo cambio científico en términos externalistas (ver Brown 1984 para algunas de las exposiciones y críticas claves). Estos son alegatos importantes que afectan a la misma razón de la enseñanza de las ciencias y que merecen la atención de los profeso. res.

Entre los educadores, el tópico sobre el pensamiento crítico - ¿qué es?, ¿cómo puede ser promovido?, ¿es transferible entre disciplinas?- ha sido asimismo un asunto muy controvertido. Está claro que una discusión sobre pensamiento crítico independiente del pensamiento científico está enormemente mutilada. Sicgel (1989) defiende la racionalidad, situándola como el punto de

\section{NOTAS}

*Traducción a cargo de Antonio Moreno González. Departamento de Didáctica de las Ciencias Experimentales. Facultad de Educación. Universioad Complutense de Madrid.

1. Este trabajo es una versión aumentada del que apareció originalmente en Studies in Science Education, 18, 1990. Se publicó ínte gramente en las actas del congreso internacional de la European Physical Society - History of The Physical Mathematical Sciences and the Teaching of Sciences"-celebrado en Madrid (Moreno 1992). Se ofrece como una visión del estado actual de los estudios sobre la relación entre la historia. la filosofía y la enseñanza de las ciencias. Esta visión es, por supuesto, parcial; otras, enfatizarán aspectos diferentes. Cubriendo, como lo hace, aspectos de la historia de la ciencia, la filosofía de la ciencia, la historia de la enseñanza de las ciencias y las corrientes actuales sobre enseñanza de las ciencias, es presumible que no se haya hecho justicia a ningún aspecto concreto.

Espero publicar pronto un trabajo siruilar en relación con la enseñanza de las matemáticas.

La investigeción fue posible gracias a la ayuda de la universidad de New South Wales. Se ha enriquecido gracias a la cooperacion ac numerosos estudiosos de todo el mundo, de los que he leido sus manuscritos para números especiales de revistas especializadas que he editado en los últimos años y de los que me he beneficiado con sus consejos y conversaciones.

2. El British National Curriculum está detatlado en NCC (1988). Se comenta en Akeroyd (1989), Solomon (1990) y Ray (1990). El Danish Curriculum en «The History and Technology of Science», se comenta en Nielsen y Nielsen (1988) y Nielsen contraste de la enseñanza de las ciencias contra el dogmatismo, por una parte, y el irracionalismo de Feyerabend, por la otra. Eger (1990) retoma la cuestión de cómo tales concepciones tienen en cuenta el papel de compromiso, o fe, que ha sido tan importante para cl desarrollo de la ciencia.

\section{CONCLUSIÓN}

He hablado de lo que considero temas emergentes en ciencia, filosofía, historia y enseñanza de las ciencias que presentan una imagen más rica y más variopinta de la ciencia de la que gencralmente es presentada en los textos escolares y en las aulas. Los nuevos currículos intentan aportar algo de esta imagen más rica al aula. El éxito dependerá, ante todo, de la introducción apropiada de cursos sobre historia y filosofía de la ciencia dirigidos tanto al profesor de ciencias en formación como al que está en ejercicio profesional. La ciencia es uno de los grandes logros de la cultura humana. La enseñanza de las ciencias, usando las palabras de un informe de 1918 de la British Association for the Advancement of Science, debería transmitir "más el espíritu y menos el resto» de este logro. Si esto se hace así, se podrán conseguir unos principios para vencer la presente crisis social e intelectual de la enseñanza de las ciencias.

y Thomsen (1990). En Holanda hay un curso sobre «Physics in Society» desde 1981 (Eijkelhof y Swager 1983); y desde 1972 varios materiales generados por el proyecto PLON (Project Curriculum Development in Physics. PO Box 80. 008. 3508 TA Utrecht) han incorporado aspectos de historia y filosofía de la ciencia. Las propuestas del proyecto 2061 están contenidas en AAAS 1989), y se comentan en Stein (1989). En McFadden (1989) se puede encontrar un comentario de los programas CTS y una guía de las publicaciones.

3. La conferencia se basó en seis revistas especializadas que contenían 55 trabajos: Educational Philosophy and Theory, 20 (2), 1988; Interchange, 20 (2), 1989; Synthese, 80 (1), 1989; Studies in Philosophy and Education, 10 (1), 1990; International Journal of Science Education, 12 (3), 1990; y Science Education, 75 (1), 1991. Se publicaron las actas en dos volúmenes (Herget 1989, 1990). Una selección de trabajos, junto con alguna otra cosa, ha sido publicada en forma de libro (Matthews 1991).

4. Para las actas de las conferencias, ver Bevilacqua y Kennedy (1983), Thomsen (1986), Blondel y Brouzeng (1988) y Moreno (1992).

5. Los escritos de Ernst Mach en el siglo xtx, sobre ensetianza de las ciencias, son tan amplios en el campo que cubren y tan estimulantes comoignorados. Se puede encontrar una introducción a sus opiniones en Matthwes (1989a).

6. Albert Einstein proporciona una buena confirmación de los punto de vista de Mach sobre la utilidad de la investigación histórica. En su ensayo autobiográfico comenta como, al final 
del siglo XIX, los físicos nunca se cansaban de intentar basar la teoría del electromagnetismo de Maxwell en principios mecánicos. Dice que «fue Ernst Mach quien, en su History of Mechanics, hizo temblar esta fe dogmática: "este libro ejerció en mí un profunda influencia a este respecto cuando yo era estudiante" (Schlipp 1951, p. 21). Éste fue el catalizador que permitió a Einstein "entar en una crítica de la mecánica comoel fundamento de la física"».

7. Schawb estuvo mucho tiempo relacionado con la Universidad de Chicago y estaba imbuido de su tradición de "grandes libros». Independientemente de Kuhn, y al mismo tiempo que El, habia enunciado una distinción entre períodos «fluidos» y «estables» de la investigación científtca, que es paralelo a la mejor conocida distinción de Kuhn entre ciencia «revolucionaria» y «normal». Se mantuvo profundamente involucrado con la teoría y la práctica de la educación, de forma comparable a la de otro filósofo-educador de Chicago, John Dewey. Una selección de sus artículos está contenida en Ford y Pugno (1964). Una lista de sus publicaciones está en Dublín (1989).

8. Se puede contactar con Brush en el Institute for Physical Science and Technology, Universidad de Maryland, College
Park, MD 20747, Estados Unjdos. Ver sus libros de 1988 para materiales de slase, y el de 1989 para un comentario sobre este campo.

9. Se puede encontrar un trabajo sobre los problemas y publicaciones de la historiografía de la ciencia en Kragh (1987). Sobre las conexiones entre la filosofía de la ciencia y la historia de la ciencia, véanse, para empezar, Giere (1973), McMullin (1975) y Wartofsky (1976).

10. Se ha disculido sobre si la ley de recapitulación es significativa para ser aplicada a materias biológicas o conceptuales. En algunos lugares, Piaget niega una recapitulación biológica, diciendo: «[... | guardémonos de volver a la idea simplista de un paralelismo nzcesario entre el đeșarrollo de la raza y el del individuo, un paralelismo que los biólogos han mostrado que es equivocado y tenđencioso» (Kitchener 1986, 6). El está más a favor de una forma conceptual de paralelismo y mantiene que en las transfornlaciones de las teorias científicas están involucrados mecanismos similares a los que hay en el cambio individual conceptual: descentralización, asimilación-acomodación, equilibrio, constructivismo, etc. Algunas de estas publicaciones se comentan en Siegel (1982)

\section{REFERENCIAS BIBLIOGRÁFICAS}

AIKENHEAD, G.S., 1980. Science in Social Issues: Implications for Teaching. (Science Council of Canada: Ottawa).

AKEROYD, F.M., 1989. Philosophy of Science in a National Curriculum, en Herget, D.E. (ed.), The History and Philosophy of Science in Science Teaching, pp. 15-22. (Florida State University).

AMERICAN ASSOCIATION FOR THE ADVANCEMENT OF SCIENCE, 1989. Science for All Americans. (AAAS: Washington).

ARONS, A.B., 1988. Historical and Philosophical Perspectives Attainable in Introductory Physics Courses, Educational Philosophy and Theory, 20(2), pp. 13-23. Reimpreso en Matthews, M.R. (ed.), Histony, Philosophy and Science Teaching: (Selected Readings. OISE Press. Toronto and Teachers College Press: Nueva York, 1991).

ASSOCIATION FOR SCIENCE EDUCATION, 1963. The Training of Graduate Science Teachers. ASE. Hatffeld, Herts.

ASSOCIATION FOR SCIENCE EDUCATION, 1979. Alternatives for Science Education. A Consultative Document. ASE. Hatfield. Herts.

ASSOCIATION FOR SCIENCE EDUCATION, 1980. What is Science? ASE. Hatfield. Herts.

ASSOCIATION FOR SCIENCE EDUCATION, 1981. Education through Science. ASE, Hatfjeld, Herts.

BAKER, G.R. y CLARK, L., 1989. The Concept of Explanation: Teaching the Philosophy of Science to Science Majors, en Herget, D.E. (ed.), The History and Philosophy of Science in Science Teaching, pp. 23-29. Florida State University.
BEVILACQL A, F., 1990. Can the History of Physics Improve Physics Teaching?, en Herget, D.E. (ed.), The History and Philosophy of Science in Science Teaching. Vol II. Florida State Univisrsity.

BEVILACQUA, F. y KENNEDY,P.J.(eds.), 1983. Proceedings of the Inter.7ational Conference on Using History of Physics in Innovatory Physics Education. (University of Pavia: Pavia).

BIRCH, C., 1988. Whitehead and Science Education, Educational Philosoph)' and Theory, 20(2), pp. 33-41.

BLEIER, R., 1384. Science and Gender. (Pergamon Press: Nueva York).

BROCK, W.H., 1987. History of Science in British Schoots: Past. Present \& Future, en Shortland, M. y Wanviek, A. (eds.), Teaching the History of Science, pp. 30-41. (Basil Blackwell: Oxford).

BROWN, J.R.(ed.), 1984. Scientific Rationality: The Sociological Turn. (Reidel: Dordrecht).

BRUMBY, MI., 1979. Problems in Learning the Concept of Natural Selection, Journal of Biological Education, 13, pp. 119-122.

BRUSH, S.G., 1974. Should the History of Science be Rated $\mathrm{X}$ ?, Science, 18, pp. 1164-1172.

BRUSH, S.G., 1988a. The History of Modern Science: A Guide to the Second Scientific Revolution. 1800-1950. Iowa State University Press. Ames 10.

BRUSH, S.G. (ed.), 1988b. History of Physics: Selected Reprints, American Association of Physics Teachers. College Park MD. 
BRUSH, S.G., 1989. History of Science \& Science Education Interchange, 20(2), pp. 60-70.

BRUSH, S.G. y KING, A.L. (eds.), 1972. History in the Teaching of Physics. (University Press of New England: Hanover, $\mathrm{NH})$.

BUCHDAHL, G., 1983. Styles of Scientific Thinking, en Bevilacqua, P.J. y Kennedy (eds.), Using History of Physics in Innovatory Physics Education, pp. 106-127. University of Pavia.

BYBEE, R.W., 1990. Teaching History and the Nature of Science in Science Courses: A Rationale, Science Education.

CAREY, S., 1986. Cognitive Psychology and Science Education, American Psychologist, 41, pp. 1123-1130.

CAWTHORN, E.R. y ROWELL, J.A., 1978. Epistemology \& Science Fducation, Studies in Science Education, 5. pp. 3159.

CHAMPAGNE, A.B., KLOPFER, L.E. y ANDERSON, J., 1980. Factors Influencing Leaming of Classical Mechanics, American Journal of Physics, 48, pp. 1074-1079.

CHAMPAGNE, A.B., GUNSTONE, R.F. y KLOPFER, L.E., 1983. Naive Knowledge and Science Learning, Research in Science and Technology Edtcation, 1(2), pp. 173-183.

CI.EMENT, J., 1983. A Conceptual Model Discussed by Gatileo and Intuitively Used by Physics Students, en Genter, D. y Stevens, A.L. (eds.), Mental Models, pp. 325-339. (Erlbaum: Hillsdale, NJ).

COHEN, I.B., 1950. A Sense of History in Science, American Journal of Physics, 18, pp. 343-359.

COHEN, I.B., 1977. History and the Philosopher of Science, en Suppes, P. (ed.), The Structure of Scientific Theories. $2^{3}$ edicion, pp. 308*360. University of Illinois Press. Urbana.

COLLINS, A., 1989. Assessing Biology Teachers: Understanding the Nature of Science and its Influence on the Practice of Teaching, en Hcrget, D.E. (ed.),The History and Philosophy of Scierice in Science Teaching, pp. 61-70. Florida State University.

CONANT, J.B., 1945. General Education in a Free Society: Report of the Harvard Committee. (Harvard University Press: Cambridge).

CONANT, J.B., 1947. On Understandings Science. (Yale University Press: New Haven).

CONANT, J.8., Science and Common Sense. (Yale University Press: New Haven).

CONANT. J.B., (ed.), 1957. Harvard Case Histories in Experinental Science, 2 vols. (Harvard University Press: Cambridge).

CONNELI.Y, F.M., 1974. Significant Connections Between Philosophy of Science and Science Education, Studies in Philosophy and Education, 8, pp. 245-2S7.

CROMBIE, A.C., 1981. Philosophical Presuppositions and the Shifting Interpretations of Galileo, en Hintilkka, J. et al. (eds.), Theory Change. Ancient Axiomatics, and Galleo's Methodology. (Reidel: Boston).
DIJKSTERHUIS, E.J., 1986. The Mechanization of the World Picture, orig. 1961. (Princeton University Press: Princeton).

DISESSA, A.A., 1982. Unlearning Aristotelian Physics: A Study of Knowledge-Based Learning, Cognitive Science, 6, pp. 37-75.

DRAKE, S., 1978. Galileo At Work. (University of Chicago Press: Chicago)

DRAKE, S., 1980. Galileo. (Oxford University Press: Oxford).

DRIVER, R., et al.(eds.), 1985. Children's Ideas in Science. (Open University Press: Milton Keynes).

DRIVER, R. y OLDHAM, V., 1986. A Constructivist Approach to Curriculum Development in Science, Studies in Science Education, 13, pp. 105-122.

DUBLIN, M., 1989. J.J. Schwab. A Memoir and a Tribute, Interchange, 20(2), pp. 112-118.

DUHEM, P., 1906/1954. The Ain \& Structure of Physical Theory. Trad. P.P. Wiener. (Princeton University Press: Princeton).

DUSCHL, R.A., 1985. Science Education \& Philosophy of Science, Twenty-five Years of Mutually Exclusive Development, School Science and Mathematics, 87(7), pp. 541-555.

DUSCHL, R.A., 1988. Abandoning the Scientistic Legacy of Science Education, Science Education, 72(1), pp. $51-62$.

DUSCHL, R.A., HAMILTON, R. y GRANDY, R.E., 1990. Psychology and Epistemology: Match or Mismatch when Applied to Science Education?, International Journalof Science Education, 12(3), pp. 230-243.

EGER,M., 1972. Physics \& Philosophy: A Problem for Education Today, American Joumal of Physics, 40, pp. 404-415.

EGER, M., 1987. Philosophy of Science in Teacher Education, en Novak, J.D. (cd.), Misconceptions \& Educational Strategies, Cornell Unjversity, Vol. I, pp. 163-176.

EGER, M., 1988. A Tale of Two Controversies: Dissonance in the Theory and Practice of Rationality, Zygon, 23(3), pp. 291326. Reimpreso en Matthews M.R. (ed.), 1991. History, Philosophy and Science Teaching: Selected Readings. (OISE Press. Toronto and Teachers College Press: Nueva York).

EGER, M., 1989. The Intercsts of Science and the Problems of Education, Synthese 80(1), pp. 81-106.

EGER, M., 1989b. Rationality and Objectivity in a Historical Approach: A Response to Harvey Sicgel, en Herget D.E. (ed.), The History and Philosophy of Science in Science Teaching, pp. 143-153. Florida State University, Tallahassce.

EIJKELHOF, H. y SWAGER, J., 1983. Physics in Society: New Trends in Physics Teaching IV. (UNESCO: París).

ENNIS, R.H., 1979. Research in Philosophy of Science Bearing on Science Education, en Asquith, P.D y Kyburg, H.E. (eds.), Current Research in Philosophy of Science, PSA, East Lansing, pp. 138-170.

FEYERABEND, P.K., 1975. Against Method. (New Left Books: Londres). 
FINOCCHIARO, M.A., 1980a. Galleo and the Ant of Reasoning. (ReideI: Dordrecht).

FINOCCHIARO, M.A. et al., 1980b. A Symposium on the Use of the History of Science in the Science Curriculum, Journal of College Science Teaching, 10(1), pp. 14-33.

FLODEN, R.E., BUCHMANN, M. y SCHWILLE, I.R., 1987. Breaking with Everyday Experience, Teachers College Record, $88(4)$, pp. $485-506$

FORGE, J.C., 1979. A Role for the Philosophy of Science in the Teaching of Science, Journal of Philosophy of Education, 13, pp. 109-118.

FURIÓ, C.J. et al., 1987. Parallels Between Adolescents' Conceptions of Gases and the History of Chemistry, Journal of Chemical Education, 64(7), pp. 616-618.

GARRISON, J. y BENTLEY, M., 1989. Science Education, Conceptual Change and Breaking with Everyday Experience, Studies in Philosophy and Education, 10(1), pp. 19-36.

GAGNÉ, R.M., 1963. The Learning Requirements for Enquiry, Journal of Research in Science Teaching, 1(2), pp. 144-153.

GIERE, R.N., 1973. History and Philosophv of Science: Intimate Relationship or Marriage of Convenience, British Journal for the Philosophy of Science, 24, pp. 282-297.

GIERE, R.N., 1987. The Cognitive Study of Science, en Nersessian, N.J. (ed.), The Process of Science, pp. 139-160. (Martinus Nijhoff: Dordrecht).

GILL, W., (ed.), 1977. Symposium on History. Philosophy \& Science Teaching, The Australian Science Teachers Journal, 23(2), pp. 4-91.

GINEV, D., 1989. Toward a New Image of Science, Studies in Philosophy and Education, 10(1), pp. 63-72.

GJERTSEN, D., 1989. Science and Philosophy. (Penguin: Harmondsworth).

GLASERSFELD, E., 1989. Cognition, Construction of Knowledge, and Teaching, Synthese, 80(1), pp. 121-140.

GOSLING, D. y MUSSCHENGA, B. (eds.), 1985. Science Education \& Ethical Values. (Georgetown University Press: Washington DC).

GOTSCHL, J., 1990. Philosophical and Scientific Conceptions of Nature and the Place of Responsibility, Intemational Joumal of Science Education, 12(3), pp. 288-296.

GRUENDER, C.D., 1989. Some Philosophical Reflections on Constructivism. en Herget, D.E. (ed.), The History and Phylosophy of Science in Science Teaching, pp. 170-176, Florida State University

HARDING,S., 1986. The Science Question in Feminism. (Comell University Press: Ithaca).

HARRE, R., 1983. History \& Philosophy of Science in the Pedagogical Process, en Home, R.W. (ed.), Science Under Scrutiny, pp. 139-157. (Reidel: Dordrecht).

HEILBRON, J.L., 1983. The Virtual Oscillator as a Guide to Physics Students Lost in Plato's Cave, en Bevilacqua y Kennedy, P.J. (eds.), Using History of Physics in Innovatory Physics Education, pp. 162-182. Pavia.
HEILBRON, J...., 1987. Applied History of Science, ISIS, 78, pp. $552-563$.

HELM, H., GI._BERT, J. y WATTS, D.M., 1985. Thought Experiments \& Physics Education. Parts I. II, Physics Education, 20, pp. 124-131, 211-217.

HELM, H. y NJVAK, J.D. (eds.), 1983. Proceedings of the Internationa! Seminar on Misconceptions in Science \& Mathematics. (Education Department, Cornell University: Ithaca).

HERGET, D.E. (ed.), 1989. The History and Philosophy of Science in sicience Teaching. (Florida State University: Tallahassee: FL).

HERGET, D.E. (ed.), 1990. More History and Philosophy of Science in science Teaching. (Florida State University. Tallahassee: FL).

HERRON, M.D , 1971. The Nature of Scientific Inquiry, School Review, 79, pp. 170-212.

HESSE, M.B., I988. Rationality in Science and Morals, Zygon, 23(3), pp. 327-332.

HODSON, D., 1982. Science, the Pursuit of Truth? Parts I. II, School Scien;e Review, 63(225), pp. 643-652; (226), pp. 2330 .

HODSON, D., .986a. Philosophy of Science and the Science Curriculum, soumal of Philosophy of Education, 20, pp. 241251.

HODSON, D., 1 1866 . Rethinking the Role \& Status of Observation in Science Ecucation, Journal of Curriculum Studies, 18(1), pp. $381-396$.

HODSON, D.,1988a. Experiments in Science and Science Teaching, Educational Philosophy and Theory, 20(2), pp. 53-66.

HODSON, D., 1988b. Toward a Philosophically More Valid Science Curriculum, Science Education, 72, pp. 19-40.

HOLTON, G., 1952. Introduction to Concepts \& Theories in Phisical Science. (Addison-Wesley: Nueva York).

HOLTON, G. e1 al., 1967. Symposium on the Project Physics Course, The Physics Teacher, 5(5), pp. 196-231.

HOLTON, G., 1975. Science. Science Teaching \& Rationality. en Hook, S. el al. (eds.), The Philosophy of rhe Curriculum. (Promethus Hooks: Buffalo).

HOLTON, G., 1978. On the Educational Philosophy of the Project Physics Course, en The Scientific Imagination: Case Studies. (Cambridge University Press: Cambridge).

HOLTON, G., 986. "A Nation At Risk» Revisited. en The Advancement of Science \& Its Burdens. (Cambridge University Press: Cambridge).

HOME, R.W. (ed.), 1983. Science Under Scrutiny: The Place of History and Philosophy of Science. (Reidel: Dordrecht).

JACOBY, B.A. y SPARGO, P.E., 1989. Ptolemy Revived?, Interchange, 20(2), pp. 33-53.

JENKINS, E., 1490. History of Science in Schools: Retrospect and Prospect in the UK, International Journal of Science 
Education, 12(3), pp. 274-281. Reimpreso en Matthews, M.R. (ed.), 1991. History Philosophy and Science Teaching: Selected Readings. OISE Press, Toronto and Teachers College Press. Nueva York 1991.

JUNG, W., 1986. Cognitive Science and the History of Science, en Thomsen, P.V. (ed.), Science Education and the History of Physics. University of Aarhus.

KAUFFMAN, G.B., 1989. History in the Chemistry Curriculum, interchange, 20(2), pp. 81-94. Reimpreso en Matthews, M.R.(ed.), History, Philosophy and Science Teaching: Selected Readings, OISE Press. Toronto and Teachers College Press, Nuevia York, I991.

KELLEK, E.F., 1985. Reflections on Gender and Science. (Yale University Press: New Haven).

KITCHER, P., 1988. The Child as Parent of the Scientist, Mind and Language, 3(3), pp. 217-228.

KITCHNER, R.F., 1985. A Bibliography of Philosophical Work on Piaget, Synthese, 65(1), pp. 139-151.

KLEIN, M.J., 1972. Use and Abuse of Historical Teaching in Physics, en Brush, S.G. y King, A.L. (eds.), History in the Teaching of Physics. (University Press of New England: Hanover).

KLOPFIR, L.E y COOLEY, W.W., 1963. The History of Science Cases for High Schools in the Development of Student Understanding of Science and Scientists: A Report on the HOSC Instruction Project, Joumal of Research in Science Teaching, 1, pp. 33-47.

KOULAIDIS, V. y OGBORN, J., 1989. Philosophy of Science: An Empirical Study of Teachers Views, Intemational Joumal of Science Education, 11(2), pp. 173-184.

KOYRÉ, A., 1939, 1978. Galileo Studies. Trad. J. Mepham, Harvester Press. (Hassocks: Sussex).

KOYRE, A., 1943. Galileo and Plato, Journal of the History of ldeas, 4, pp. 400-428. (Reproducido en Metaphysics and Measurement, 1968.)

KOYRÉ, A., 1986. Metaphysics and Measurement. (Harvard University Press: Cambridge).

KRAGH, H., 1987. An Introduction to the Historiography of Science. (Cambridge University Press: Cambridge).

KRAJEWSKI, W. (ed.), 1982. Polish Essays in the Philosophy of the Natural Sciences. (Reidel: Dordrecht),

KRASILCHIK, M., 1990. The «Scientists»: A Brazilian Experiment in Science Education, International Journal of Science Education, 12(3), pp. 282-287.

KUHN, T.S., 1959. The Essential Tension: Tradition and Innovation in Scientific Research The Third University of Utah Research, Conference on the Identification of Scientific Talent. University of Utah Press. Salt Lake City. Reimpreso en The Essential Tension, pp. 225-239. (University of Chicago Press: Chicago).

KUHN, T.S., 1977. Concepts of Cause in the Development of Physics, en The Essential Tension, pp. 21-30. (University of Chicago Press: Chicago).

LAKATOS, I., 1978. History of Science and its Rational
Reconstructions. en, Worrall y Currie, G. (eds.), The Methodology of Scientific Research Programmes, pp. 102138. (Cambridge University Press: Cambridge)

LEDERMAN, N.G. y ZEIDLER, D.L., 1987. Science Teachers Conceptions of the Nature of Science: Do They Really Influence Teaching Behaviour?, Science Education, 7 1(5), pp. 721-734

LIPMAN, M. y SHARP, A.M., (eds.), 1978. Growing Up with Philosophy. (Temple University Press: Philadelphia).

LOVING, C.C., 1992. The Scientific Theory Profile: A Philosophy of Science Model for Science Teachers, Journal of Research in Science Teaching.

MACH, E., 1883, 1960. The Science of Mechanics, Open Court Publishing Company, LaSalle Il.

MACH, E., 1895, 1943. On Instruction in the Classics \& the Sciences, en his Popular Scientific Lectures, Open Court, LaSalle.

MACHAMER, P., 1978. Galileo and the Causes, en Butts, R.E. y Pitt, J.C. (eds.), New Perspectives on Galileo, pp. 161-181. Dordrecht. Reidel.

MAcLACHLAN, J., 1990. Drake Against the Philosophers, en Levere, T.H. y Shea, W.R. (eds.), Nature, Experiment, and the Sciences, pp. 123-144. Kiuwer, Dordrecht.

MANUEL, D.E., 1981. Reflections on the Role of History \& Philosophy of Science in School Science Education, School Science Review, 62(221), pp. 769-771.

MARKUS, G., 1987. Why Is There No Hermeneutics of Natural Sciences: Some Preliminary Theses, Science in Context, 1(1), pp. 5-51.

MARTIN, J.R., 1989. Ideological Critiques and the Philosophy of Science, Philosophy of Science, 56, pp. 1-22.

MARTIN, J.R., What Should Science Educators Do About the Gender Bias in Science?, en Matthews, M.R. (ed.), History, Philosophy and Science Teaching, pp. 151-166. Selected Readings, OISE Press, Toronto and Teachers College Press, Nueva York, 1991

MARTIN, M., 1972. Concepts of Science Education, (Scott, Foresman \& Co: Nueva York) (Reimpreso en University Press of America, 1985).

MARTIN, M, 1986. Science Education \& Moral Education, Journal of Moral Education, 15(2), pp. 99-108. Reimpreso en Matthews, M.R. (ed.), History, Philosophy and Science Teaching: Selected Readings, OISE Press, Toronto and Teachers College Press, Nueva York 1991.

MATTHEWS, M.R., 1987. Galileo's Pendulum \& the Objects of Science, en Arnstine B. y D. (eds.), Philosophyof Education, pp. 309-319, Philosophy of Education Society.

MATTHEWS, M.R., 1988. A Role for History and Philosophy in Science Teaching, Educational Philosophy and Theory, 20(2), pp. 67-81.

MATTHEWS, M.R., 1989a. Ernst Mach and Thought Experiments in Science Education, Research in Science Education, 18, pp. $251-258$

MATTHEWS, M.R., (ed.), 1989b. The Scientific Background 
to Modern Philosophy. (Hackett Publishing Company: Indianapolis).

MATTHEWS, M.R., 1990a. History, Philosophy, and Science Teaching: The Case of Pendulum Motion, Research in Science Education, 19, pp. 187-198.

MATTHEWS, M.R., 1990b. History, Philosophy, and Science Teaching: What Can Be Done in an Undergraduate Course? Studies in Philosophy and Education, 10(1), pp. 93-98.

MATTHEWS, M.R., (ed.), 1991.History, Philosophy, and Science Teaching: Selected Readings. (OISE Press: Toronto).

MCCLOSKEY, M., 1983. Intuitive Physics, Scientific American, 248, pp. 114-122.

MCDERMOTT, L.C., 1984. Research on Conceptual Understanding in Mechanics, Physics Today, 37, pp. 24-32.

McMULLIN, E., 1975. History and Philosophy of Science: a Marriage of Convenience?, Boston Studies in the Philosophy of Science, 32, pp. 515-531.

MCTIGHE, T.P., 1967. Gailiteo's Platonism: A Reconsideration, en McMutlen, E. (ed.), Galileo: Man of Science, pp. 365 388. (Basic Books: Nueva York).

MENDELSOHN, E., 1976. Values and Science: a Critical Reassessment, The Science Teacher, 43(1), pp. 20-23.

MERTON, R.K.,1977. The Sociology of Science: An Episodic Memoir, en Merton, R.K. y Gaston, J. (eds.), The Sociology of Science in Europe, pp. 3-141. (Southem Illinois University Press, Carbondale: IL).

MILLER, J.D., 1983. Scientific Literacy: A Conceptual \& Empirical Review, Daedalus, 112(2), pp. 29-47.

MISCHEL, T., 1971. Piaget: Cognitive Conflict and the Motivation of Thought, en Mischel, T. (ed.), Cognitive Development and Epistemology, pp. 311-355. Nueva York.

MITTELSTRASS, J., 1972. The Galilean Revolution: The Historical Fate of a Methodological Insight, Studies in the History and Philosophy of Science, 2, pp. 297-328.

MORENO, A., (ed.), 1992. Proceedings of the International Conference on History of the Physical. Mathematical Sciences and the teaching of Sciences, European Physical Society. (Universidad Complutense de Madrid: Madrid).

MURRAY, F.R., (ed.), 1979. The Impact of Piagetian Theory on Education. Philosophy, Psychiatry, and Psychology. Baltimore.

NATIONAL CURRICULUM COUNCIL, 1988. Science in the National Curriculum. (NCC: York).

NERSESSIAN, N.J.. 1989. Conceptual Change in Science and in Science Education, Synthese, 80(1), pp. 163-184. Reimpreso en, Matthews, M.R.(ed.), 1991,History, Philosophyand Science Teaching: Selecled Readings, OISE Press. (Toronto and Teachers College Press: Nueva York).

NIELSEN, H. y THOMSEN, P., 1990. History and Philosophy of Science in the Danish Curriculum, International Journal of Science Education, 12(3), pp. 308-316.

NORRIS, S.P., 1985. The Philosophical Basis of Observation in Science and Science Education, Journal of Research in
Science Teaching, 22(9), pp. 817-833.

NOVAK, J.D., 1987. Human Constructivism: Toward a Unity of Psychological \& Epistemological Meaning Making, en Novak, J.D. (ed.), 1987, Misconceptions \& Educationol Strategies, Vcl I, pp. 349-360.

NOVAK, J.D. (ed.), 1987. Proceedings of the Second Intemational Seminar on Misconceptions \& Educational Strategies in Science, \& Mathematics. (Education Department. Cornell University: It]jaca).

NOVAK, L., 1980. The Structure of Idealization. (ReideI: Dordrecht).

NUSSBAUM, J.. 1983. Classroom Conceptual Change: The Lesson to be Learned from the History of Science, en Helm, H. y Novak, J.D. (eds.), Misconceptions in Science \& Mathematics, 1p. 272-281. (Department of Education. Comell University: Itjuaca).

PASSMORE, J., 1978. Science and its Critics. (Duckworth: Londres).

PIAGET, J., 197C. Genetic Epistemology. (Columbia University Press: Londres).

PIAGET, J. y GA.RCÍA, R., 1989. Psychogenesis and History. (Columbia Uriversity Press: Nueva York).

POSNER, G. et al., 1982. Accommodation of a Scientific Conception: Tijwards a Theory of Conceptual Change, Science Education, 66(2), pp. 211-227.

PUMFREY, S., 987. The Concept of Oxygen: Using History of Science in \$cience Teaching, en Shortland, M. y Warwick, A. (eds.), Teaching the History of Science, pp. 142-155. (Basil Blackwell: O;ford).

RANDALL, J.H., 1940. The School of Padua and the Emergence of Modern Science, Journal of the History of Ideas, 1, pp. 177-206. Reirnpreso en The Career of Philosophy. Volume One, 1961. (Columbia University Press: Nueva York).

ROBIN, N. y OHLSSON, S., 1989. Impetus Then and Now: A Detailed Comparison between Jean Buridan and a Single Contemporary Subject, en Hegert, D.E. (ed.), The History and Philosopiy of Science Teaching, pp. 292-305. Florida State University.

ROHRLICH, F, 1988. Four Philosophical Issues Essential for Good Science Teaching, Educational Philosophy and Theory, 20(2), pp. 1-6.

ROWELL, J.A., 1989. Piagetian Epistemology: Equilibration and the Teacl ing of Science, Synthese, 80(1), pp. 141-162.

ROWELL, I.A. y CAWTHRON, E.R., 1982. Images of Science: An Empirical ștudy, European Joumal of Science Education, 4(1), pp. 79-94.

RUSSELL, T.L., 1981. What History of Science, How Much and Why?, Science Education, 65, pp. 51-64.

SCHECKER, H., 1988. The Paradigmatic Change in Mechanics: Implications if Historical Processes on Physics Education, en Blondel, C. y Brouzeng, P. (eds.), Science Education and the History of Physics, pp. 215-220.

SCHEFFLER, I, 1970. Philosophy and the Curriculum, en Reason and Teaching, 1973, pp. 31-44. (Routledge: Londres). 
SCHILPP, P.A. (ed.), 1951. Albert Einstein, $2^{a}$ ed. (Tudor: Nueva York).

SCHWAB, J.J., 1963. Biology Teachers Handbook. (Wiley: Nueva York).

SCHWAB, J., 1964. Structure of the Disciplines: Meaning \& Significances, en Ford, G.W. y Pugno, L. (eds.), The Structure of Knowledge \& the Curriculum. (Rand McNally \& Co.. Chicago).

SIEGEl, H., 1979. On the Distortion of the History of Science in Science Education, Science Education 63, pp. 111-118.

SIEGEI,, H., 1982. On the Parallel between Piagetian Cognitive Development \& the History of Science, Philosophy of Social Science, 12, pp. 375-386

SETTLE, T., 1990. How to Avoid Implying that Physicalism is True: A Problem for Teachers of Science, International Journal of Science Education, 12(3), pp. 258-264.

SHAPERE, D., 1984. Reason and the Search for Knowledge, (Reidel: Dordrecht).

SHEA, W.R., 1972. Galileo's Intellectiual Revolution. (Macmillan: Lontires).

SHERRATT, W.J., 1982. History of Science in the Science Curriculum: An Historical Perspective, School Science Review 64, pp. 225-236, 418-424.

SHORTLAND, M. y WARWICK, A. (ed's.), 1989. Teaching the History of Science. (Basil Blaclcwell: Oxford).

SHUELL, T. 1987.Cognitive Psychology and Conceptual Change: Implications for Teaching Science, Science Education, 71, pp. $239-250$.

SHULMAN, L.S., 1986. Those Who Understand: Knowledge Growth in Teaching, Educational Researcher, 15(2), pp. 4-14.

SHULMAN, L.S., 1987. Knowledge and Teaching: Foundations of the New Reform, Harvard Educational Review, 57(1), pp. 22.

SIEGEI,, H., 1987. Relativism Refuted. (Reidel. Dordrecht).

SIEGEL., H., 1989. The Rationality of Science. Critical Thinking, and Science Education, Synthese, 80(1), pp. 9-42. Reimpreso en Matthews, M.R. (ed.) 1991, History, Philosophy and Science Teaching: Selected Readings. OLSE Press. Toronto and Teachers Coliege Press. Nueva York, 1991.

SOLOMON, J., 1990. Teaching About the Nature of Science in the British National Curriculum, Science Education.

STEIN, F., 1989. Project 2061: Education for a Changing Future. en Herget, D.E. (ed.), The History and Philosophy of Science in Science Teaching, pp. 339-343. Florida State University.

STEINBERG, M.S., BROWN., D.E y CLEMENT, J., Genius is not Immune to Persistent Misconceptions: Conceptual Difficulties Impeding Isaac Newton and Contemporany Physics Students, International Journal of Science Education, 12(3), pp. 256-273.

STEVENS, P., 1978. On the Nuffield Philosophy of Science, Journal of Philosophy of Education, 12, pp. 99-111.
STINNER, A., 1990. Philosophy. Thought Experiments, and Large Context Problems in the Secondary School Physics Course, International Journal of Science Education, 12(3), pp. $244-257$.

STRIKE, K.A., 1987. Towards a Coherent Constructivism, en Novak, J.D. (ed.), Misconceptions \& Educational Strategies, Education Department. Cornell University. Vol. I. pp. 481489.

SUCHTING, W.A., 1992. Constructivism Deconstructed, Science \& Education, $1(3)$.

SUMMERS, M.K., 1982. Philosophy of Science in the Science Teacher Education Curriculum, European Joumal of Science Education, 4, pp. 19-28.

SWIFT, J.N., 1988. The Tyranny of Terminology: Biology, The Science Teachers Bulletin, 60(2), pp. 24-26.

TAMIR, P., 1989. History and Phijosophy of Science and Biological Education in Israel, Interchange, 20(2), pp. 95 98 .

THAYER, H.S., (ed.), 1953. Newton's Philosophy of Nature. (Macmilian: Nueva York).

THOMSON, J.J., 1918. Natural Science in Education, HMSO. Londres. (Known as the Thomson Report).

THOMSEN, P.V. (ed.), 1986. Science Education and the History of Physics, University of Aarhus.

TOULMIN, S.E., 1983. The Construal of Reality: Criticism. in Modern \& Post-Modern Science, en Mitchell, W.J.T. (ed.), The Politics of Interpretation. University of Chicago Press, Chicago, pp. 99-117. (Original, 1982, Critical Inquiry, pp. 93- \11.

VIENNOT, L., 1979. Spontaneous Reasoning in Elementary Dynamics, European Journal of Science Education, 1, pp. 205-221.

WAGENSCHEIN, M., 1962. Die Padagogische Dimension der Physik, Westermans, Braunschweig.

WALLACE, W.A., 1981. Galileo and Reasoning ex suppositione, en Wallace, W.A. Prelude to Galileo, pp. 129-159. (Reidel: Dordrecht).

WALLACE, W.A., 1984. Galileo and His Sources. (Princeton University Press: Princeton).

WANDERSEE, J.H., 1985. Can the History of Science Help Science Educators Anticipate Students' Misconceptions?, Journal of Research in Science Teaching, 23(7), pp. 581597.

WARTOFSKY,M., 1968. Metaphysics as a Heuristic for Science, en Cohen, R.S. y Wartofsky M.W. (eds.), Boston Studies in the Philosophy of Science, 3, pp. 123-172. Reimpreso en Models, 1979, pp. 40-89. (Reidel: Dordrecht).

WARTOFSKY, M.W., 1976. The Relation Between Philosophy of Science and History of Science, en Cohen, R.S., Feyerabend, P.K. y Wartofsky, M.W. (eds.), Essays in Memory of Imre Lakatos. (Reidel: Dordrecht). (Boston Studies in the Philosophy of Science, Vol. 39). Reimpreso en Models, 1979. (Reidel: Dordrecht).

WELCH, W., 1979. Twenty Years of Science Education 


\section{HISTORIA Y EPISTEMOLOGÍA DE LAS CIENCIAS}

Development: A Look Back, Review of Research in Education 7 , pp. 282-306.

WHEWELL, W., 1840, 1947. Philosophy of the Inductive Sciences. Londres.

WHITAKER, M.A.B., 1979. History and Quasi-history in Physics Education, I. II, Physics Education 14, pp. 108-112, 239242.

WHITAKER, R.I., 1983. Aristotle is Not Dead: Student Understanding of Trajectory Motion, American Joumal Physics, 51(4), pp. 352-357.
WINCHESTER, I., 1990. Thought Experiments and Conceptual Revision in Science, Studies in Philosophy and Education, $10(1)$, pp. 73-\$0.

WOOLNOUGH, B.E., 1989. Faith in Science, School Science Review, 70(2:2), pp. 133-137. Reimpreso en Matthews, M.R. (ed.), History, Philosophy and Science Teaching: Selected Readings. OISE Press. Toronto and Teachers College Press. Nueva York 1991.

ZIMAN, J., 1980. Teaching and Learning about Science and Society. (Camoridge University Press: Cambridge). 
